\title{
Adaptive Fixed-Time 6-DOF Coordinated Control of Multiple Spacecraft Formation Flying with Input Quantization
}

\author{
Shiyu Wang, ${ }^{1}$ Ruixia Liu $\mathbb{D}^{2,3}$ and Lihua Wen ${ }^{1}$ \\ ${ }^{1}$ School of Astronautics, Northwestern Polytechnical University, Xian 710072, China \\ ${ }^{2}$ School of Automation, Xi'an University of Posts and Telecommunications, Xian 710072, China \\ ${ }^{3}$ Xi'an Key Laboratory of Advanced Control and Intelligent Process, Xi'an University of Posts and Telecommunications, \\ Xian 710072, China \\ Correspondence should be addressed to Ruixia Liu; ruixialiu@xupt.edu.cn
}

Received 10 October 2020; Revised 23 November 2020; Accepted 9 December 2020; Published 30 December 2020

Academic Editor: Jing $\mathrm{Na}$

Copyright (c) 2020 Shiyu Wang et al. This is an open access article distributed under the Creative Commons Attribution License, which permits unrestricted use, distribution, and reproduction in any medium, provided the original work is properly cited.

\begin{abstract}
This paper investigates the fixed-time coordinated control problem of six-degree-of-freedom (6-DOF) dynamic model for multiple spacecraft formation flying (SFF) with input quantization, where the communication topology is assumed directed. Firstly, a new multispacecraft nonsingular fixed-time terminal sliding mode vector is derived by using neighborhood state information. Secondly, a hysteretic quantizer is utilized to quantify control force and torque. Utilizing such a quantizer not only can reduce the required communication rate but also can eliminate the control chattering phenomenon induced by the logarithmic quantizer. Thirdly, a 6-DOF fixed-time coordinated control strategy with adaptive tuning laws is proposed, such that the practical fixed-time stability of the controlled system is ensured in the presence of both upper bounds of unknown external disturbances. It theoretically proves that the relative tracking errors of attitude and position can converge into the regions in a fixed time. Finally, a numerical example is exploited to show the usefulness of the theoretical results.
\end{abstract}

\section{Introduction}

The recent decades have seen an ever increasing research interest in the coordinated control problem of spacecraft formation flying (SFF) due to its successful applications in the space industry such as atmosphere monitoring of the Earth, deep space exploration, and spacecraft on-orbit maintenance [1-5]. As is well known, the coordinated control of attitude and orbit are two equally important technologies. It is essential to achieve the desired attitude and position simultaneously for SFF mission [6, 7]. Owning to the dynamical coupling between orbit motion and attitude motion, these two motions can be considered as a whole six-degree-of-freedom (6-DOF) motion. Recently, the 6DOF coordinated control of SFF has attracted considerable research attention $[8,9]$. However, the control strategies proposed in the aforementioned literature can only guarantee asymptotic stability of the controlled systems [10-13].
For coordinated control problems of SFF, fast convergence performance is an important requirement [14-18]. In contrast to asymptotic stabilization controllers, the finitetime stabilization controllers can provide a faster response and better disturbance-rejection ability [19-22]. Therefore, the finite-time controllers have been developed in spacecraft formation control [23-25]. Even though the finite-time control methods can ensure the controlled systems finitetime stabilization, the convergence time relies on the information of initial system states, which gives rise to difficulties in practical applications [26]. To cope with this constraint, the fixed-time stable concept was applied to study finite-time controller design, in that the convergence time is upper bounded regardless of initial system states [27-29]. To date, fixed-time control strategies have been used for various control systems $[30,31]$ but less attention has been paid to fixed-time 6-DOF coordinated control problem for SFF, especially for external disturbances with unknown upper 
bounds. Another significant issue in multiple SFF task is that the interspacecraft communication links are not always bidirectional, such as in the unidirectional spacecraft laser communication system. However, in some of the existing results, the coordinated control issue is investigated based on an assumption that the communication topology is undirected.

On another research frontier, networked control systems (NCSs) as an active field of research have been applied successfully in various modern complicated engineering processes [13, 32-35] such as unmanned vehicles, nuclear power stations, and aerospace engineering systems. In modern low-cost plug-and-play small spacecraft formation systems, the functional components connected by wireless networked media [36-39]. It is quite common that when the signal is transmitted between the control and actuator module via wireless networks, the SFF systems unavoidably suffer from quantization errors caused by quantization behavior which will degrade the control performance or even lead to instability [40-43]. Thus, it is needed to propose new controllers for SFF where the signal quantization is taken into consideration. Although some research attention has been centered on the quantized control problem of SFF, there is still no result available that considers 6-DOF coordinated control of multiple spacecraft formation in the presence of quantized input control signal. The complexity of the multiple spacecraft formation coordinated control task makes the quantized fixed-time coordinated control a serious challenge.

In this paper, we are motivated to deal with the problem of fixed-time 6-DOF adaptive coordinated control for multiple SFF with input quantization under directed communication topology. The main contributions of this paper are highlighted as follows: (1) the communication topology among follower spacecraft is described by a directed graph, which will bring more challenges than the case that the communication topology among follower spacecraft is described by an undirected graph. (2) A novel multispacecraft nonsingular FTTSM based on a 6-DOF dynamic model is designed, on which each spacecraft converges to its desired states while keeping synchronization with other formation spacecraft. (3) A fixed-time adaptive coordinate control strategy is derived to compensate for the effects of hysteretic quantizer and external disturbances on the control performance and guarantee the practical fixed-time stability of the controlled system.

The rest of this paper is organized as follows: in section 2, the modelling and preliminaries are presented. In section 3, a multispacecraft nonsingular fixed-time terminal sliding mode vector is designed. In section 4, a fixed-time adaptive coordinated control scheme is proposed. An illustrative example and a conclusion are given in Sections 5 and 6, respectively.

\section{Modelling and Preliminaries}

2.1. 6-DOF Dynamic Model. The 6-DOF dynamic model of spacecraft formation is represented as follows [8]:

$$
\left\{\begin{array}{l}
\dot{\mathrm{x}}_{1 i}=\Lambda\left(\dot{\mathrm{x}}_{1 i}\right) \mathbf{x}_{2 i}, \\
\mathbf{G}_{f i} \dot{\mathrm{x}}_{2 i}+\mathbf{C}\left(\mathbf{x}_{2 i}\right)+\mathbf{N}\left(\mathbf{x}_{1 i}\right)+\boldsymbol{\tau}_{i}=\mathbf{u}_{i}, \quad i=1,2, \ldots, n,
\end{array}\right.
$$

where

$$
\begin{aligned}
\mathbf{x}_{1 i} & =\left[\begin{array}{l}
\boldsymbol{\rho}_{i} \\
\mathbf{q}_{i}
\end{array}\right], \mathbf{x}_{2 i}=\left[\begin{array}{l}
\dot{\rho}_{i} \\
\boldsymbol{\omega}_{i}
\end{array}\right], \boldsymbol{\Lambda}\left(\dot{\mathbf{x}}_{1 i}\right)=\left[\begin{array}{cc}
\mathbf{I}_{3 \times 3} & 0_{3 \times 3} \\
0_{3 \times 3} & \frac{\mathbf{T}\left(\mathbf{q}_{i}\right)}{2}
\end{array}\right], \mathbf{G}_{f i}=\left[\begin{array}{cc}
m_{f i} \mathbf{I}_{3 \times 3} & 0_{3 \times 3} \\
0_{3 \times 3} & \mathbf{J}_{f i}
\end{array}\right], \\
\mathbf{C}\left(\mathbf{x}_{2 i}\right) & =\left[\begin{array}{c}
\mathbf{C}\left(n_{0}\right) \dot{\rho}_{i} \\
\boldsymbol{\omega}_{i}^{\times} \mathbf{J}_{f i} \boldsymbol{\omega}_{i}
\end{array}\right], \mathbf{N}\left(\mathbf{x}_{1 i}\right)=\left[\begin{array}{c}
N\left(\boldsymbol{\rho}_{i}, n_{0}, R\right) \\
0_{3 \times 1}
\end{array}\right], \boldsymbol{\tau}_{i}=\left[\begin{array}{c}
\mathbf{F}_{d i} \\
\mathbf{z}_{i}
\end{array}\right], \mathbf{u}_{i}=\left[\begin{array}{c}
\mathbf{u}_{f i} \\
\mathbf{u}_{t i}
\end{array}\right], \\
\mathbf{T}\left(\mathbf{q}_{i}\right) & =\left[\begin{array}{c}
-\mathbf{q}_{v i}^{T} \\
q_{0 i} \mathbf{I}_{3 \times 3}+\mathbf{q}_{v i}^{\times}
\end{array}\right], C\left(n_{0}\right)=2 n_{0}\left[\begin{array}{ccc}
1 & 0 & 0 \\
0 & 0 & 0
\end{array}\right], N\left(\boldsymbol{\rho}_{i}, n_{0}, R\right)=\left[\begin{array}{c}
-\dot{n}_{0} y_{i}-n_{0}^{2} x_{i}-2 \frac{\mu}{R^{3}} x_{i} \\
\dot{n}_{0} x_{i}-n_{0}^{2} y_{i}+\frac{\mu}{R^{3}} y_{i} \\
\frac{\mu}{R^{3}} z_{i}
\end{array}\right],
\end{aligned}
$$

where superscript $i$ stands for the $i$ th follower spacecraft; $\rho_{i}=\left[\begin{array}{ll}x_{i} & y_{i} z_{i}\end{array}\right]^{T}$ represents the relative position vector from the $i$ th follower spacecraft to the leader spacecraft; $\omega_{i} \in \mathbb{R}^{3}$ denotes the angular velocity; $\mathbf{q}_{i} \in \mathbb{R}^{4}$ is the quaternion defined as $\mathbf{q}_{i}=\left[\begin{array}{ll}q_{0 i} & \mathbf{q}_{v i}\end{array}\right]^{T}$, where $n_{0} \in \mathbb{R}$ represents angular velocity of the virtual leader spacecraft; $q_{0 i}$ is the scalar part and $\mathbf{q}_{v i}$ is the vector part; $m_{f i} \in \mathbb{R}$ denotes the mass; $\mathbf{J}_{f i} \in \mathbb{R}^{3 \times 3}$ is the inertia matrix; $\mathbf{u}_{f i} \in \mathbb{R}^{3}$ represents the control force; $\mathbf{u}_{i t} \in \mathbb{R}^{3}$ is the control torque; $\mathbf{F}_{d i} \in \mathbb{R}^{3}$ is disturbance force; and $\mathbf{z}_{i}=\ell_{i}-\mathbf{T}_{i G T}$, where $\ell_{i} \in \mathbb{R}^{3}$ is 
disturbance torque and $\mathbf{T}_{i G T} \in \mathbb{R}^{3}$ is the gravity gradient torque. The notation $1^{\times}$for the vector $\imath=\left[\begin{array}{lll}l_{1} & l_{2} & l_{3}\end{array}\right]^{T}$ represents the skew-symmetric matrix as follows:

$$
l^{\times}=\left[\begin{array}{ccc}
0 & -l_{3} & l_{2} \\
l_{3} & 0 & -l_{1} \\
-l_{2} & l_{1} & 0
\end{array}\right] .
$$

It is worth to mention that the attitude and orbit are mutually coupled by $\mathbf{T}_{i G T} \in \mathbb{R}^{3}$, which is given as

$$
\mathbf{T}_{i G T}=3 \mu \frac{\widehat{\mathbf{R}}_{f i}^{\times} \mathbf{J}_{f i} \widehat{\mathbf{R}}_{f i}}{\left(x_{i}^{2}+\left(R+y_{i}\right)^{2}+z_{i}^{2}\right)^{(3 / 2)}},
$$

where $\widehat{\mathbf{R}}_{f i} \in \mathbb{R}^{3}$ is the position unit vector. Since $\mathbf{T}_{i G T}$ is much smaller compared with control torque, $\mathbf{T}_{i G T}$ is always treated as a disturbance.

We define the following error states:

$$
\mathbf{e}_{1 i}=\left[\begin{array}{c}
\boldsymbol{\rho}_{i}-\boldsymbol{\rho}_{d i} \\
\mathbf{q}_{e i}
\end{array}\right], \mathbf{e}_{2 i}=\left[\begin{array}{c}
\dot{\boldsymbol{\rho}}_{e i} \\
\boldsymbol{\omega}_{e i}
\end{array}\right]=\left[\begin{array}{c}
\dot{\rho}_{i}-\dot{\rho}_{d i} \\
\boldsymbol{\omega}_{i}-R\left(\mathbf{q}_{e i}\right) \boldsymbol{\omega}_{d i}
\end{array}\right],
$$

where $\mathbf{q}_{e i}$ is error quaternion defined as $\mathbf{q}_{e i}=\left[\begin{array}{cc}q_{0 e i} & \mathbf{q}_{v e i}^{T}\end{array}\right]^{T}=\mathbf{q}_{e i} \otimes \overline{\mathbf{q}}_{d i} ; R\left(\mathbf{q}_{e i}\right)$ is the rotation matrix from the $i$ th follower spacecraft's reference frame to its bodyfixed frame; and $\rho_{d i}, \dot{\rho}_{d i}, \omega_{d i}$, and $\mathbf{q}_{d i}$ are the desired position, desired velocity, desired angular velocity, and desired attitude, respectively.

Then the 6-DOF relative error dynamic model of SFF can be expressed by

$$
\left\{\begin{array}{l}
\dot{\mathbf{e}}_{1 i}=\Lambda\left(\dot{\mathbf{e}}_{1 i}\right) \mathbf{e}_{2 i}, \\
\mathbf{G}_{f i} \dot{\mathbf{e}}_{2 i}+\mathbf{C}\left(\mathbf{e}_{2 i}\right)+\mathbf{N}\left(\mathbf{e}_{1 i}\right)+\boldsymbol{\tau}_{i}=\mathbf{u}_{i},
\end{array}\right.
$$

where

$$
\begin{aligned}
\boldsymbol{\Lambda}\left(\dot{\mathbf{e}}_{1 i}\right) & =\left[\begin{array}{cc}
\mathbf{I}_{3 \times 3} & 0_{3 \times 3} \\
0_{3 \times 3} & \frac{\mathbf{T}\left(\mathbf{q}_{e i}\right)}{2}
\end{array}\right], \mathbf{N}\left(\mathbf{e}_{1 i}\right)=\left[\begin{array}{c}
N\left(\boldsymbol{\rho}_{i}, n_{0}, R\right)-\ddot{\rho}_{d i} \\
0_{3 \times 1}
\end{array}\right], \\
\mathbf{C}\left(\mathbf{e}_{2 i}\right) & =\left[\begin{array}{c}
C\left(n_{0}\right) \dot{\rho}_{i} \\
-\boldsymbol{\omega}_{i}^{\times} \mathbf{J}_{f i} \boldsymbol{\omega}_{i}+\mathbf{J}_{f i}\left(\boldsymbol{\omega}_{e i}^{\times} \boldsymbol{\omega}_{d i}-R\left(\mathbf{q}_{e i}\right) \dot{\omega}_{d i}\right)
\end{array}\right] .
\end{aligned}
$$

2.2. Graph Theory. It is supposed that the information flow among $n$ follower spacecraft is described by a directed graph $\mathrm{G}=(\mathrm{V}, \chi, \mathrm{A})$, where $\mathrm{V}=\left\{\mathrm{V}_{1}, \mathrm{~V}_{2}, \ldots, \mathrm{V}_{n}\right\}$ represents the set of nodes, $\chi \subseteq \mathrm{V} \times \mathrm{V}$ represents the set of edges, and $\left(\mathrm{V}_{i}, \mathrm{~V}_{j}\right) \in \chi$ represents if and only if node $\mathrm{V}_{i}$ can receive the information of node $\mathrm{V}_{j}$. In spacecraft 6DOD coordinated control application, $\left(\mathrm{V}_{i}, \mathrm{~V}_{j}\right) \in \chi$ represents only the $j$ th spacecraft can obtain the $i$ th spacecraft's states information. $\mathrm{A}=\left[a_{i j}\right] \in \mathbb{R}^{n \times n}$ denotes the weighted adjacency matrix of the graph $G$ with entries

$$
\begin{cases}a_{i j}>0, & \text { if }\left(\left(\mathrm{V}_{i}, \mathrm{~V}_{j}\right) \in \chi\right), \\ a_{i j}=0, & \text { otherwise, }\end{cases}
$$

where $a_{i j}$ is the nonnegative element of A, which denotes communication quality between the $i$ th spacecraft and $j$ th spacecraft. It is noticeable that self-edges are not allowed, meaning that $a_{i i}=0$.

The in-degree matrix of the graph $G$ is $\mathbf{D}$ with entries

$$
\mathbf{D}=\operatorname{diag}\left\{d_{1}, d_{2}, \ldots, d_{n}\right\}
$$

where

$$
d_{i}=\sum_{j=1}^{n} a_{i j}=\sum_{j \in \chi_{i}} a_{i j}, \quad(i=1,2, \ldots, n) .
$$

The Laplacian matrix $\mathbf{L} \in \mathbb{R}^{n \times n}$ of the graph $G$ is [44]

$$
\mathbf{L}=\mathbf{D}-A .
$$

2.3. Hysteretic Quantizer. To eliminate the control chattering phenomenon induced by logarithmic quantizer, a hysteretic quantizer is used to quantify control torque and force in this paper, which is similar to [45]. It can be expressed by

$$
q(u(t)) \triangleq \begin{cases}u_{J} \operatorname{sgn}(u), & \text { if } \frac{u_{J}}{1+\delta}<|u| \leq \frac{u_{J}}{1-\delta}, \dot{u}_{J}<0, \text { or, } \\ & u_{J}<|u| \leq \frac{u_{J}}{1-\delta}, \dot{u}_{J}>0, \\ u_{J}(1+\delta) \operatorname{sgn}(u), & \text { if } u_{j}<|u| \leq \frac{u_{J}}{1-\delta}, \dot{u}_{J}<0, \text { or, } \\ & \frac{u_{J}}{1-\delta} \leq|u| \leq \frac{u_{J}(1+\delta)}{1-\delta}, \dot{u}_{J}>0, \\ 0, & \text { if } 0 \leq|u|<\frac{u_{\min }}{1+\delta}, \dot{u}_{J}<0, \text { or, } \\ & \frac{u_{\min }}{1+\delta} \leq|u| \leq u_{\min }, \dot{u}_{J}>0, \\ & \text { othercase, }\end{cases}
$$

where $u_{J}=\rho^{(1-i)} u_{\min }, J=1,2, \ldots, n$ with $0<\rho<1, u_{\min }>0$, and $\delta=(1-\rho / 1+\rho) . \quad q(u(t)) \quad$ is in the set $U=\left\{0, \pm u_{i}, \pm u_{i}(1+\delta)\right\}$. The map of the hysteresis quantizer $q(u(t))$ for $u>0$ is illustrated in Figure 1.

Remark 1. The parameter $\rho$ can be termed as a measure of quantization density. From the definition of $\rho$, we can see that the smaller parameter $\rho$ is, the coarser the hysteretic quantizer becomes [45]. Therefore, the design of parameter $\rho$ 


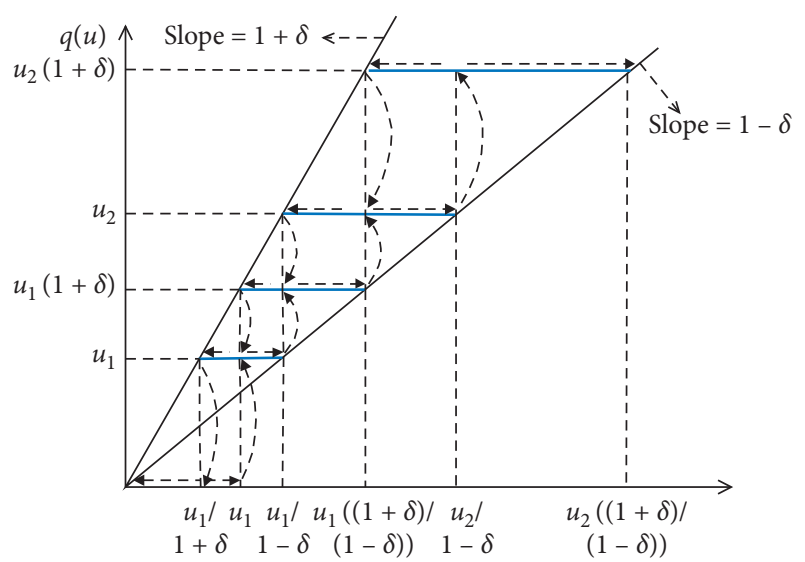

Figure 1: Map of $q(u(t))$ for $u>0$.

should be based on a criterion to guarantee the control performance with a smaller quantization density. In addition, compared with a traditional logarithmic quantizer, the hysteretic quantizer (10) can avoid oscillations by adding additional quantization levels.

2.4. Preliminaries. For deriving the 6-DOF fixed-time coordinated controller, the lemmas are made as follows.

Lemma 1. The hysteretic quantizer $q(u(t))$ is decomposed into two parts as

$$
q(u(t))=D(u) u(t)+Q(t),
$$

where $D(u)$ and $Q(t)$ satisfy

$$
1-\delta \leq D(u) \leq 1+\delta,|Q(t)| \leq u_{\min } .
$$

The proof of Lemma 1 is similar to Theorem 1 in [45].

Lemma 2 (see [44]). If $\mathrm{G}$ is a directed graph with $\mathrm{N}$ nodes, then all the eigenvalues of the weighted Laplace matrix $\mathbf{L}$ have nonnegative real part.
Lemma 3 (see [46]). For any matrix $M \in \mathbb{R}^{m \times m}, N \in \mathbb{R}^{n \times n}$, $X \in \mathbb{R}^{m \times m}$, and $Y \in \mathbb{R}^{n \times n}$, then the following equalities hold:

(1) $(M \otimes N)(X \otimes Y)=M X \otimes N Y$

(2) If the matrices $M$ and $N$ are invertible, then $(M \otimes N)^{-1}=M^{-1} \otimes N^{-1}$

(3) If the eigenvalues of $M$ are $\lambda_{1}, \ldots, \lambda_{m}$ and the eigenvalues of $N$ are $\mu_{1}, \ldots, \mu_{n}$, then the eigenvalues of $M \otimes N$ can be expressed by $\lambda_{i} \mu_{j}(i=1, \ldots, m ; j=1, \ldots, n)$

Lemma 4 (see [47]). For any $x, y \in \mathbb{R}$, if $\nu \in \mathbb{R}^{+}$and $\nu>1$, then

$$
|x+y|^{\nu} \leq 2^{\nu-1}\left|x^{\nu}+y^{\nu}\right|
$$

Lemma 5 (see [48]). If $x_{i} \in \mathbb{R}, i=1,2, \ldots, n$, and $0<p \leq 1$, then

$$
\left(\sum_{i=1}^{n}\left|x_{i}\right|\right)^{p} \leq \sum_{i=1}^{n}\left|x_{i}\right|^{p} \leq n^{1-p}\left(\sum_{i=1}^{n}\left|x_{i}\right|\right)^{p} .
$$

Lemma 6 (see [47]). Consider the nonlinear system given by

$$
\dot{x}=f(x, t), f(0, t)=0, \quad x \in \mathbb{R}^{n} .
$$

Suppose that there exists a Lyapunov function $V(x)$ that satisfies the following condition:

$$
\dot{V}(x) \leq-\left(\alpha V(x)^{p}+\beta V(x)^{g}\right)^{k}+v, \quad x \in U_{o},
$$

where $\alpha, \beta, p, g \in \mathbb{R}^{+}, p k<1, g k>1$, and $0<v<\infty$. Then, the origin of system (17) is practical fixed-time stable and the residual set of the solution satisfies

$$
\left\{\lim _{t \rightarrow T} x \mid V(x) \leq \min \left\{\alpha^{-(1 / p)}\left(\frac{v}{1-\theta k}\right)^{(1 / k p)}, \beta^{-(1 / g)}\left(\frac{v}{1-\theta k}\right)^{(1 / k g)}\right\}\right\}
$$

where $\theta$ is a scalar satisfying $0<\theta \leq 1$. The setting time is bounded by

$$
T \leq \frac{1}{\alpha^{k} \theta^{k}(1-p k)}+\frac{1}{\beta^{k} \theta^{k}(g k-1)} .
$$

\section{Multispacecraft Nonsingular Fixed-Time Terminal Sliding Mode}

In this section, a multispacecraft nonsingular fixed-time terminal sliding mode (FTTSM) vector is proposed to realize the orbit and attitude coordinated control for SFF. The following assumptions are presented regarding the 6-DOF dynamic model.

Assumption 1. The total disturbance $\tau_{i}$ is assumed to be bounded due to the fact that magnetic forces, $J_{2}$ perturbations, gravitation, and solar radiation pressure are bounded.

Assumption 2. The desired angular velocity $\omega_{d i}$ and its time derivative $\dot{\omega}_{d i}$ are assumed to be bounded. The desired trajectory $\rho_{d i}$ and its time derivative $\dot{\rho}_{d i}$ are assumed to be bounded. 
In order to achieve coordinated control for multiple spacecraft formation, the sliding mode based on (6) is defined as

$$
\mathbf{S}=\left[\mathbf{s}_{1}, \ldots, \mathbf{s}_{i}\right]^{T},
$$

where $\mathbf{s}_{i}=\left[s_{i, 1}, s_{i, 2}, \ldots, s_{i, k}\right]^{T} \in \mathbb{R}^{6 \times 1}, \quad i=1,2, \ldots, n$ and $k=1,2, \ldots, 6$, given by

$$
\mathbf{s}_{i}=b_{i} \mathbf{G}_{f i}\left[\mathbf{e}_{2 i}+\alpha_{i}\left(\mathbf{e}_{1 i}\right)\right]+\sum_{j=1}^{n} a_{i j}\left[\left(\mathbf{G}_{f i} \mathbf{e}_{2 i}-\mathbf{G}_{f j} \mathbf{e}_{2 j}\right)+\left(\mathbf{G}_{f i} \alpha_{i}\left(\mathbf{e}_{1 i}\right)-\mathbf{G}_{f j} \alpha_{j}\left(\mathbf{e}_{1 j}\right)\right)\right]
$$

with $\alpha_{i}\left(\mathbf{e}_{1 i}\right)=\left[\alpha_{i, 1}\left(e_{1 i 1}\right), \alpha_{i, 2}\left(e_{1 i 2}\right), \ldots, \alpha_{i, k}\left(e_{1 i k}\right)\right]^{T} \in \mathbb{R}^{6 \times 1}$,

and

$$
\alpha_{i, k}\left(e_{1 i k}\right)=\left\{\begin{array}{l}
\operatorname{sgn}\left(\sigma_{1 i} \operatorname{sgn}\left(e_{1 i k}\right)^{p_{1}}+\sigma_{2 i} \operatorname{sgn}\left(e_{1 i k}\right)^{p_{2}}\right)^{k_{1}}, \quad \text { if } \bar{s}_{i, k}=0 \text { or } \bar{s}_{i, k} \neq 0,\left|e_{1 i k}\right|>\varepsilon \\
l_{1 i} e_{1 i k}+l_{2 i} \operatorname{sgn}\left(e_{1 i k}\right)^{2}, \quad \text { if } \bar{s}_{i, k} \neq 0,\left|e_{1 i k}\right| \leq \varepsilon
\end{array}\right.
$$

where $i=1, \ldots, n, k=1,2, \ldots, 6, \quad \bar{s}_{i}=\left[\bar{s}_{i, 1}, \bar{s}_{i, 2}, \ldots, \bar{s}_{i, 6}\right]^{T}$, $\bar{s}_{i}=\mathbf{e}_{2 i}+\operatorname{sgn}\left(\sigma_{1 i} \operatorname{sgn}\left(\mathbf{e}_{1 i}\right)^{p_{1}}+\sigma_{2 i} \operatorname{sgn}\left(\mathbf{e}_{1 i}\right)^{p_{2}}\right)^{k_{1}}, l_{1 i}=\left(2-k_{1}\right)$ $\left(\sigma_{1 i} \varepsilon^{p_{1}-\left(1 / k_{1}\right)}+\sigma_{2 i} \varepsilon^{p_{2}-\left(1 / k_{1}\right)}\right)^{k_{1}}, \quad l_{2 i}=\left(k_{1}-1\right)\left(\sigma_{1 i} \varepsilon^{p_{1}-\left(1 / k_{1}\right)}\right.$ $\left.+\sigma_{2 i} \varepsilon^{p_{2}-\left(1 / k_{1}\right)}\right)^{k_{1}}, b_{i}>0$ is control scheme gain to realize tracking control, $a_{i j} \geq 0$ is control scheme gain to realize coordinated control between $i$ th and $j$ th formation spacecraft, $\operatorname{sgn}\left(e_{1 i k}\right)^{p_{1}}=$ $\left|e_{1 i k}\right|^{p_{1}} \operatorname{sgn}\left(e_{1 i k}\right)$ and $\operatorname{sgn}\left(e_{1 i k}\right)^{p_{2}}=\left|e_{1 i k}\right|^{p_{2}} \operatorname{sgn}\left(e_{1 i k}\right), \sigma_{1 i}>0$, $\sigma_{1 i}>0, \quad p_{1}>0, \quad p_{2}>0 k_{1}>0$ are designed parameters, $\operatorname{sgn}\left(\sigma_{1 i} \operatorname{sgn}\left(e_{1 i k}\right)^{p_{1}}+\sigma_{2 i} \operatorname{sgn}\left(e_{1 i k}\right)^{p_{2}}\right)^{k_{1}}=\mid\left(\sigma_{1 i} \operatorname{sgn}\left(e_{1 i k}\right) \quad p_{1}+\right.$ $\left.\sigma_{2 i} \operatorname{sgn}\left(e_{1 i k}\right)^{p_{2}}\right)\left.\right|^{k_{1}} \operatorname{sgn}\left(\sigma_{1 i} \operatorname{sgn}\left(e_{1 i k}\right)^{p_{1}}+\sigma_{2 i} \operatorname{sgn}\left(e_{1 i k}\right)^{p_{2}}\right), \varepsilon>0$ is small constant, $0<p_{1} k_{1}<1, p_{2} k_{1}>1$, and $\operatorname{sgn}(\cdot)$ is sign function.

Remark 2. It is supposed that the communication topology of SFF is undirected; we can obtain $a_{i j}=a_{j i}$, which simplifies the design and analysis of the controller. However, for the directed communication topology, $a_{i j}=a_{j i}$ does not hold. Therefore, compared with bidirectional communication topology, the coordinated control of formation spacecraft under directed communication topology is more challenging.

Remark 3. Note that the multispacecraft FTTSM (21) can be simplified as a modified terminal sliding mode (TSM) designed in [47] if $k_{1}=1$ and $p_{2}=0$; moreover, (21) coincides with the modified fast TSM designed in [49] for $k_{1}=1$. It is worth mentioning that when $e_{1 i k}$ converges to the region $\left|e_{1 i k}\right| \leq \varepsilon$, the multispacecraft FTTSM is converted to the general sliding mode for $\bar{s}_{i, 1} \neq 0$. Thus, the singularity problem of (21) can be effectively avoided. Moreover, by the choice of $l_{1}$ and $l_{2}$, the continuity of $\alpha_{i, k}$ and its first-order time derivative is guaranteed.
By Kronecker product, the sliding mode function (21) can be described by

$$
\mathbf{S}=\left[(\mathbf{L}+\mathbf{B}) \otimes \mathbf{I}_{6}\right] \overline{\mathbf{G}}\left(\mathbf{e}_{2}+\alpha\left(\mathbf{e}_{1}\right)\right),
$$

where $\mathbf{L}$ is the weighted Laplace matrix, which is determined by directed topology, $\mathbf{B}=\operatorname{diag}\left[b_{1}, b_{2}, \ldots, b_{n}\right], \mathbf{e}_{2}=$ $\left[\mathbf{e}_{21}^{T}, \mathbf{e}_{22}^{T}, \ldots, \mathbf{e}_{2 n}^{T}\right]^{T}, \quad \alpha\left(\mathbf{e}_{1}\right)=\left[\alpha_{1}^{T}\left(\mathbf{e}_{11}\right), \alpha_{2}^{T} \quad\left(\mathbf{e}_{12}\right), \ldots, \alpha\right.$ $\left.{ }_{n}^{T}\left(\mathbf{e}_{1 n}\right)\right]^{T}, \overline{\mathbf{G}}=\operatorname{diag}\left[\mathbf{G}_{f 1}, \mathbf{G}_{f 2}, \ldots, \mathbf{G}_{f n}\right]$.

\section{Design of Fixed-Time Adaptive Coordinated Control Scheme}

In this section, a fixed-time adaptive 6-DOF coordinated control scheme is presented for multiple SFF with input quantization and external disturbances.

To design the control scheme, (6) can be derived as

$$
\mathbf{G}_{f i}\left(\dot{e}_{2 i}+\dot{\alpha}_{i}\left(\mathbf{e}_{1 i}\right)\right)=\mathbf{h}_{i}+D\left(u_{i}\right) \mathbf{u}_{i}+Q_{i}(t)+\boldsymbol{\tau}_{i},
$$

with

$$
\begin{aligned}
& \dot{\alpha}_{i}\left(\mathbf{e}_{1 i}\right)=\left\{\begin{array}{l}
\sigma_{1 i} p_{1} k_{1} \operatorname{diag}\left(\left|\sigma_{1 i} \operatorname{sgn}\left(\mathbf{e}_{1 i}\right)^{p_{1}}+\sigma_{2 i} \operatorname{sgn}\left(\mathbf{e}_{1 i}\right)^{p_{2}}\right|^{k_{1}-1}\right), \\
\cdot \operatorname{diag}\left(\left|\mathbf{e}_{1 i}\right|^{p_{1}-1}\right) \boldsymbol{\Lambda}\left(\dot{e}_{1 i}\right) \mathbf{e}_{2 i}+\sigma_{2 i} p_{2} k_{1} \operatorname{diag}\left(\mid \sigma_{1 i} \operatorname{sgn}\left(\mathbf{e}_{1 i}\right)^{p_{1}},\right. \\
\left.+\left.\sigma_{2 i} \operatorname{sgn}\left(\mathbf{e}_{1 i}\right)^{p_{2}}\right|^{k_{1}-1}\right) \operatorname{diag}\left(\left|\mathbf{e}_{1 i}\right|^{p_{2}-1}\right) \boldsymbol{\Lambda}\left(\dot{e}_{1 i}\right) \mathbf{e}_{2 i}, \\
\text { if } \bar{s}_{i k}=0 \text { or } \bar{s}_{i k} \neq 0,\left|e_{1 i k}\right|>\varepsilon \\
l_{1 i} \boldsymbol{\Lambda}\left(\dot{x}_{1 i}\right) \mathbf{e}_{2 i}+2 l_{2 i} \operatorname{diag}\left(\left|e_{1 i}\right|\right) \boldsymbol{\Lambda}\left(\dot{e}_{1 i}\right)\left(\mathbf{e}_{2 i}\right), \\
\text { if } \bar{s}_{i k} \neq 0,\left|e_{1 i k}\right| \leq \varepsilon
\end{array}\right. \\
& \mathbf{h}_{i}=-\mathbf{C}\left(\mathbf{e}_{2 i}\right)-\mathbf{N}\left(\mathbf{e}_{1 i}\right)-\mathbf{G}_{f i}-\dot{e}_{2 i d}+\mathbf{G}_{f i} \dot{\alpha}_{i}\left(\mathbf{e}_{1 i}\right) .
\end{aligned}
$$


Under Assumption 1, it can be seen that

$$
\left(\left\|(\mathbf{L}+\mathbf{B}) \otimes \mathbf{I}_{6}\right\|_{1}\left\|\boldsymbol{\tau}_{i}+Q_{i}(t)\right\|_{1}\right)^{2} \leq c_{i}, \quad i=1, \ldots, n,
$$

where $c_{i}$ are nonnegative constant numbers.

The fixed-time adaptive controller is designed as

$$
\begin{aligned}
& \mathbf{u}_{i}=-\frac{1}{1-\delta} \mathbf{h}_{i}+\frac{1}{1-\delta}\left(\sum_{j=1, j \neq i}^{n} a_{i j}+b_{i}\right)^{-1}\left[\sum_{j=1, j \neq i}^{n} a_{i j}\left((1-\delta) \mathbf{u}_{j}+\mathbf{h}_{j}\right)-\alpha_{i} \operatorname{sgn}\left(\mathbf{s}_{i}\right)^{\gamma 1},-\beta_{i} \operatorname{sgn}\left(\mathbf{s}_{i}\right)^{\gamma 2}-\frac{\widehat{c}_{i}}{2 \mathfrak{J}_{i}^{2}} \mathbf{s}_{i}\right], \quad i=1, \ldots, n, \\
& \dot{\hat{c}}_{i}=-2 \kappa_{i} \varrho_{i} \widehat{c}_{i}+\frac{\kappa_{i}}{2 \mathfrak{J}_{i}^{2}}\left\|s_{i}\right\|, \quad i=1, \ldots, n,
\end{aligned}
$$

where $0<\gamma 1<1, \gamma 2>1, \mathfrak{\Im}_{i}>0, \kappa_{i}>0$, and $\varrho_{i}>0$ are the controller parameters.

$$
\left\|s_{i}\right\| \leq \Delta_{s}=\min \left\{v_{1}^{-(2 / 1+\gamma 1)}\left(\frac{v}{1-\theta_{o}}\right)^{(2 / 1+\gamma 1)},\left(v_{2}\left(\frac{1}{2^{v+12-1}}\right)^{n+k-1}\right)^{-(2 / 1+\gamma 1)}\left(\frac{v}{1-\theta_{o}}\right)^{(2 / 1+\gamma 2)}\right\}
$$

in fixed time, where $\beta_{\text {min }}=\min \left\{\beta_{i}\right\}, \quad \alpha_{\min }=\min \left\{\alpha_{i}\right\}$, $\iota_{\min }=\min \left\{\iota_{i}\right\}, \quad v_{1}=\min \left\{\alpha_{\min } 2^{(\gamma 1+1 / 2)}, \iota_{\min }(\gamma 1+1 / 2) 2^{(\gamma 1+1 / 2)}\right\}$, $v_{2}=\min \left\{\beta_{\min } 2^{(\gamma 2+1 / 2)}, \iota_{\min (\gamma 2+1 / 2)} 2^{(\gamma 2+1 / 2)}\right\}, \quad v=\sum_{i=1}^{n} \varrho_{i} o_{i} c_{i}^{2}$ $+1+\sum_{i=1}^{n} / \mathfrak{J}^{2} 2+\sum_{i=1}^{n}\left[\left(\left(\iota_{i} / \kappa_{i}\right) \Delta_{i}^{2}\right)^{(\gamma 2+1 / 2)}-\left(\iota_{i} / \kappa_{i}\right) \Delta_{i}^{2}\right]$ $\iota_{i}=\kappa_{i}\left(\varrho_{i}\left(2 o_{i}-1\right) / 2 o_{i}\right), \Delta_{i}>0,0<\theta_{0} \leq 1, o_{i}>(1 / 2)$.

Proof. We construct the following Lyapunov function candidate:

$$
V_{1}=V_{2}+V_{3}
$$

with

$$
V_{2}=\frac{1}{2} \mathbf{S}^{T} \mathbf{S}, V_{3}=\frac{1}{2} \sum_{i=1}^{n} \kappa_{i}^{-1} \widetilde{c}_{i}^{2}
$$

where $\widetilde{c}_{i}=c_{i}-\widehat{c}_{i}$.

By the Kronecker product, the controller (28) can be rewritten as

$$
\begin{aligned}
\mathbf{U}= & -\frac{1}{1-\delta} \mathbf{H}+\frac{1}{1-\delta}\left[(\mathbf{D}+\mathbf{B})^{-1} \otimes \mathbf{I}_{6}\right] \\
& {\left[\left(\mathbf{A} \otimes \mathbf{I}_{6}\right)((1-\delta) \mathbf{U}+\mathbf{H})-\boldsymbol{\alpha} \operatorname{sgn}(\mathbf{S})^{\gamma 1},\right.} \\
& -\boldsymbol{\beta} \operatorname{sgn}(\mathbf{S})^{\gamma 2}-\widehat{c} \mathbf{S} .
\end{aligned}
$$

where $\quad \mathbf{U}=\left[\mathbf{u}_{1}, \ldots, \mathbf{u}_{n}\right]^{T}, \quad \mathbf{H}=\left[h_{1}, \ldots, h_{n}\right]^{T}$, $\alpha=\operatorname{diag}\left[\alpha_{1} \mathbf{I}_{6}, \ldots, \alpha_{n} \mathbf{I}_{6}\right], \quad \alpha=\operatorname{diag}\left[\alpha_{1} \mathbf{I}_{6}, \ldots, \alpha_{n} \mathbf{I}_{6}\right], \widehat{c}$ $=\operatorname{diag}\left[\left(\widehat{c}_{1} / 2 \mathfrak{J}_{1}^{2}\right) \mathbf{I}_{6}, \ldots,\left(\widehat{c}_{n} / 2 \mathfrak{J}_{n}^{2}\right) \mathbf{I}_{6}\right]$.

Since $\mathbf{I}_{6 n}-\left[(\mathbf{D}+\mathbf{B})^{-1} \otimes \mathbf{I}_{6}\right]\left(\mathbf{A} \otimes \mathbf{I}_{6}\right)$ can be equivalently expressed as
Theorem 1. Consider the 6-DOF control system (6) with the fixed-time coordinated control law (28). If the parameter uncertainty and external disturbance satisfy Assumptions 1-2, then the sliding mode vector $\mathbf{s}_{i}$ will converge into

$$
\begin{aligned}
& \mathbf{I}_{6 n}-\left[(\mathbf{D}+\mathbf{B})^{-1} \otimes \mathbf{I}_{6}\right]\left(\mathbf{A} \otimes \mathbf{I}_{6}\right), \\
& \left.=\left[(\mathbf{D}+\mathbf{B})^{-1} \otimes \mathbf{I}_{6}\right]\left\{(\mathbf{D}+\mathbf{B}) \otimes \mathbf{I}_{6}\right]-\mathbf{A} \otimes \mathbf{I}_{3}\right\}, \\
& =\left[(\mathbf{D}+\mathbf{B})^{-1} \otimes \mathbf{I}_{6}\right]\left[(\mathbf{L}+\mathbf{B}) \otimes \mathbf{I}_{6}\right] .
\end{aligned}
$$

It follows from (34) that

$$
\begin{aligned}
\mathbf{U}= & -\frac{1}{1-\delta} \mathbf{H}-\frac{1}{1-\delta}\left\{\mathbf{I}_{6 n}-\left[(\mathbf{D}+\mathbf{B})^{-1} \otimes \mathbf{I}_{6}\right]\left(\mathbf{A} \otimes \mathbf{I}_{6}\right)\right\}^{-1} \\
& {\left[(\mathbf{D}+\mathbf{B})^{-1} \otimes \mathbf{I}_{6}\right], }
\end{aligned}
$$

$$
\begin{aligned}
& \times\left(\boldsymbol{\alpha} \operatorname{sgn}(\mathbf{S})^{\gamma 1}+\boldsymbol{\beta} \operatorname{sgn}(\mathbf{S})^{\gamma 2}+\widehat{c} \mathbf{S}\right) \\
= & -\frac{1}{1-\delta} \mathbf{H}-\frac{1}{1-\delta}\left\{\left[(\mathbf{D}+\mathbf{B})^{-1} \otimes \mathbf{I}_{6}\right]\left[(\mathbf{L}+\mathbf{B}) \otimes \mathbf{I}_{6}\right]\right\}^{-1} \\
& {\left[(\mathbf{D}+\mathbf{B})^{-1} \otimes \mathbf{I}_{6}\right] } \\
& \times\left(\boldsymbol{\alpha} \operatorname{sgn}(\mathbf{S})^{\gamma 1}+\boldsymbol{\beta} \operatorname{sgn}(\mathbf{S})^{\gamma 2}+\widehat{c} \mathbf{S}\right), \\
= & -\frac{1}{1-\delta} \mathbf{H}-\frac{1}{1-\delta}\left[(\mathbf{L}+\mathbf{B}) \otimes \mathbf{I}_{6}\right]^{-1}
\end{aligned}
$$$$
\left(\boldsymbol{\alpha} \operatorname{sgn}(\mathbf{S})^{\gamma 1}+\boldsymbol{\beta} \operatorname{sgn}(\mathbf{S})^{\gamma 2}+\widehat{c} \mathbf{S}\right)
$$

Considering (24) and (25), it can be shown that 


$$
\begin{aligned}
\dot{V}_{2} & =\mathbf{S}^{T} \dot{\boldsymbol{S}}, \\
& =\mathbf{S}^{T}\left[(\mathbf{L}+\mathbf{B}) \otimes \mathbf{I}_{6}\right]\left(\mathbf{G}_{f i} \dot{\mathrm{e}}_{2 i}+\mathbf{G}_{f i} \dot{\alpha}_{i}\left(\mathbf{e}_{1 i}\right)\right), \\
& =\mathbf{S}^{T}\left[(\mathbf{L}+\mathbf{B}) \otimes \mathbf{I}_{6}\right](\mathbf{H}+\mathbf{D}(U) \mathbf{U}+\mathbf{Q}(t)+\boldsymbol{\tau}),
\end{aligned}
$$

where $\tau=\left[\tau_{1}, \ldots, \tau_{n}\right]^{T}, \mathbf{D}(U)=\operatorname{diag}\left[\mathbf{D}\left(u_{1}\right), \ldots, \mathbf{D}\left(u_{n}\right)\right]$, $\mathbf{Q}(t)=\left[Q_{1}(t), \ldots, Q_{n}(t)\right]^{T}$.

Next, it can be derived that

$$
\begin{aligned}
& \dot{V}_{2}=\mathbf{S}^{T}\left[(\mathbf{L}+\mathbf{B}) \otimes \mathbf{I}_{6}\right]\left(\left[(\mathbf{L}+\mathbf{B}) \otimes \mathbf{I}_{6}\right]^{-1}\left(-\boldsymbol{\alpha} \operatorname{sgn}(\mathbf{S})^{\gamma 1}-\boldsymbol{\beta} \operatorname{sgn}(\mathbf{S})^{\gamma 2}-\mathbf{c S}\right)+Q(t)+\boldsymbol{\tau}\right), \\
& =\mathbf{S}^{T}\left[(\mathbf{L}+\mathbf{B}) \otimes \mathbf{I}_{6}\right](\boldsymbol{\tau}+Q(t))-\mathbf{S}^{T} \boldsymbol{\alpha} \operatorname{sgn}\left(S^{\gamma 1}-\mathbf{S}^{T} \boldsymbol{\beta} \operatorname{sgn}\left(\mathbf{S}^{\gamma 2}-\mathbf{S}^{T} \widehat{c} \mathbf{S},\right.\right. \\
& \leq \sum_{i=1}^{n}\left\|(\mathbf{L}+\mathbf{B}) \otimes \mathbf{I}_{6}\right\|\left\|\boldsymbol{\tau}_{i}+Q_{i}(t)\right\|\left\|\mathbf{s}_{i}\right\|-\sum_{i=1}^{n} \frac{\widehat{c}_{i}}{2 \mathfrak{\Im}_{i}^{2}}\left\|\mathbf{s}_{i}\right\|^{2}-\sum_{i=1}^{n} \sum_{k=1}^{p} \beta_{i}\left|s_{i k}\right|^{\gamma 2+1}, \\
& -\sum_{i=1}^{n} \sum_{k=1}^{p} \alpha_{i}\left|s_{i k}\right|^{\gamma 1+1} \\
& \leq \sum_{i=1}^{n} \frac{\left\|\left((\mathbf{L}+\mathbf{B}) \otimes \mathbf{I}_{6}\left\|_{1}\right\| \boldsymbol{\tau}_{i}+Q_{i}(t) \|_{1}\right)^{2}\right\| \mathbf{s}_{i} \|^{2}}{2 \mathfrak{J}^{2}}+\sum_{i=1}^{n} \frac{\mathfrak{J}^{2}}{2}-\sum_{i=1}^{n} \frac{\widehat{c}_{i}}{2 \mathfrak{J}_{i}^{2}}\left\|\mathbf{s}_{i}\right\|^{2}, \\
& -\sum_{i=1}^{n} \sum_{k=1}^{p} \alpha_{i}\left(s_{i k}^{2}\right)^{(\gamma 1+1 / 2)}-\sum_{i=1}^{n} \sum_{k=1}^{p} \beta_{i}\left(s_{i k}^{2}\right)^{(\gamma 1+1 / 2)}, \\
& \leq \sum_{i=1}^{n} \frac{\widetilde{c}_{i}}{2 \mathfrak{\Im}_{i}^{2}}\left\|\mathbf{s}_{i}\right\|^{2}+\sum_{i=1}^{n} \frac{\mathfrak{J}^{2}}{2}-\sum_{i=1}^{n} \sum_{k=1}^{p} \alpha_{i}\left(s_{i k}^{2}\right)^{(\gamma 1+1 / 2)}-\sum_{i=1}^{n} \sum_{k=1}^{p} \beta_{i}\left(s_{i k}^{2}\right)^{(\gamma 2+1 / 2)} .
\end{aligned}
$$

In addition, taking the derivative of $V_{3}$ yields

$$
\begin{aligned}
\dot{V}_{3} & =\sum_{i=1}^{n} \kappa_{i}^{-1} \widetilde{c}_{i} \dot{\tilde{c}}_{i}, \\
& =-\sum_{i=1}^{n} \frac{\widetilde{c}_{i}}{2 \widetilde{\mathfrak{J}}_{i}^{2}}\left\|\mathbf{s}_{i}\right\|^{2}+\sum_{i=1}^{n} 2 \varrho_{i} \widetilde{c}_{i} \widehat{c}_{i} .
\end{aligned}
$$

It is noticed from (37) and (38) that

$$
\begin{aligned}
\dot{V}_{1} \leq & -\sum_{i=1}^{n} \sum_{k=1}^{p} \alpha_{i}\left|s_{i k}\right|^{\gamma 1+1}-\sum_{i=1}^{n} \sum_{k=1}^{p} \beta_{i}\left|s_{i k}\right|^{\gamma 2+1} \\
& +2 \sum_{i=1}^{n} \varrho_{i} \widetilde{c}_{i} \widehat{c}_{i}+\sum_{i=1}^{n} \frac{\mathfrak{J}^{2}}{2}
\end{aligned}
$$

From

$$
\varrho_{i} \widetilde{c}_{i} \widehat{c}_{i} \leq-\frac{\varrho_{i}\left(2 o_{i}-1\right)}{2 o_{i}} \widetilde{c}_{i}^{2}+\frac{\varrho_{i} o_{i}}{2} c_{i}^{2}
$$

and by substituting (40) into (39), we have

$$
\begin{aligned}
\dot{V}_{1} \leq & -\sum_{i=1}^{n} \sum_{k=1}^{p} \alpha_{i}\left|s_{i k}\right|^{\gamma 1+1}-\sum_{i=1}^{n} \sum_{k=1}^{p} \beta_{i}\left|s_{i k}\right|^{22+1}-\sum_{i=1}^{n} \frac{\underline{t}_{i}}{\kappa_{i}} \widetilde{c}_{i}^{2}+\sum_{i=1}^{n} \varrho_{i} o_{i} c_{i}^{2}, \\
& -\sum_{i=1}^{n} \frac{\iota_{i}}{\kappa_{i}} \widetilde{c}_{i}^{2}-\sum_{i=1}^{n}\left(\frac{\underline{i}_{i}}{\kappa_{i}} \widetilde{c}_{i}^{2}\right)^{\left(1+\gamma_{1} / 2\right)}+\sum_{i=1}^{n}\left(\frac{\underline{l}_{i}}{\kappa_{i}} \widetilde{c}_{i}^{2}\right)^{\left(1+\gamma_{1} / 2\right)}+\sum_{i=1}^{n} \frac{\widetilde{\mathfrak{J}}^{2}}{2} .
\end{aligned}
$$

where $\left(\iota_{i} / \kappa_{i}\right)=\left(\varrho_{i}\left(2 o_{i}-1\right) / 2 o_{i}\right)$.

Case 1. If $\left(\iota_{i} / \kappa_{i}\right) \widetilde{c}_{i}^{2} \geq 1$, we have

$$
\left(\frac{l_{i}}{\kappa_{i}} \widetilde{c}_{i}^{2}\right)^{\left(1+\gamma_{1} / 2\right)} \leq \frac{l_{i}}{\kappa_{i}} \widetilde{c}_{i}^{2}
$$

Substituting (42) into (41) yields

$$
\begin{aligned}
\dot{V}_{1} \leq & -\sum_{i=1}^{n} \sum_{k=1}^{p} \alpha_{i}\left(s_{i k}^{2}\right)^{(\gamma 1+1 / 2)}-\sum_{i=1}^{n} \sum_{k=1}^{p} \beta_{i}\left(s_{i k}^{2}\right)^{(\gamma 2+1 / 2)} \sum_{i=1}^{n} \frac{l_{i}}{\kappa_{i}} \widetilde{c}_{i}^{2} \\
& +\sum_{i=1}^{n} \varrho_{i} o_{i} c_{i}^{2}-\sum_{i=1}^{n}\left(\frac{\iota_{i}}{\kappa_{i}} \widetilde{c}_{i}^{2}\right)^{(\gamma 1+1 / 2)}+\sum_{i=1}^{n} \frac{\mathfrak{J}^{2}}{2} .
\end{aligned}
$$

According to Lemma 5, it is easy to prove that 


$$
\sum_{i=1}^{n} \sum_{k=1}^{p} \alpha_{i}\left(s_{i k}^{2}\right)^{(\gamma 1+1 / 2)}+\sum_{i=1}^{n}\left(\frac{i_{i}}{\kappa_{i}} \widetilde{c}_{i}^{2}\right)^{(1+\gamma 1 / 2)} \geq v_{1} V_{8}^{(1+\gamma 1 / 2)}
$$

where $\quad v_{1}=\min \left\{\alpha_{\min } 2^{(\gamma 1+1 / 2)}, \iota_{\min }(\gamma 1+1 / 2) 2^{(\gamma 1+1 / 2)}\right\}$, $\alpha_{\min }=\min \left\{\alpha_{i}\right\}, \iota_{\min }=\min \left\{l_{i}\right\}$.

By applying (44), (43) can be rewritten as

$$
\begin{aligned}
\dot{V}_{1} \leq & -v_{1} V_{1}^{(1+\gamma 1 / 2)}-\sum_{i=1}^{n} \sum_{k=1}^{p} \beta_{i}\left(s_{i k}^{2}\right)^{(\gamma 2+1 / 2)}-\sum_{i=1}^{n} \frac{\iota_{i}}{\kappa_{i}} \widetilde{c}_{i}^{2} \\
& +\sum_{i=1}^{n} \mathrm{e}_{i} o_{i} c_{i}^{2}+\sum_{i=1}^{n} \frac{\mathfrak{\Im}^{2}}{2}
\end{aligned}
$$

Case 2. If $\left(\iota_{i} / \kappa_{i}\right) \widetilde{c}_{i}^{2}<1$, one can obtain

$$
\left(\frac{l_{i}}{\kappa_{i}} \widetilde{c}_{i}^{2}\right)^{\left(1+\gamma_{1} / 2\right)}-\frac{l_{i}}{\kappa_{i}} \widetilde{c}_{i}^{2} \leq 1-\frac{l_{i}}{\kappa_{i}} \widetilde{c}_{i}^{2}<1 .
$$

Substituting (46) into (41) yields

$$
\begin{aligned}
\dot{V}_{1} \leq & -v_{1} V_{1}^{(1+\gamma 1 / 2)}-\sum_{i=1}^{n} \sum_{k=1}^{p} \beta_{i}\left(s_{i k}^{2}\right)^{(\gamma 2+1 / 2)}-\sum_{i=1}^{n} \frac{\iota_{i}}{\kappa_{i}} \widetilde{c}_{i}^{2} \\
& +\sum_{i=1}^{n} \varrho_{i} o_{i} c_{i}^{2}+1+\sum_{i=1}^{n} \frac{\mathfrak{J}^{2}}{2} .
\end{aligned}
$$

Furthermore, (47) can be rewritten as

$$
\begin{aligned}
\dot{V}_{1} \leq & -v_{1} V_{1}^{(1+\gamma 1 / 2)}-\sum_{i=1}^{n} \sum_{k=1}^{p} \beta_{i}\left(s_{i k}^{2}\right)^{(\gamma 2+1 / 2)} \\
& -\sum_{i=1}^{n} \frac{\iota_{i}}{\kappa_{i}} \widetilde{c}_{i}^{2}-\sum_{i=1}^{n}\left(\frac{\iota_{i}}{\kappa_{i}} \widetilde{c}_{i}^{2}\right)^{(\gamma 2+1 / 2)}, \\
& +\sum_{i=1}^{n}\left(\frac{\iota_{i}}{\kappa_{i}} \widetilde{c}_{i}^{2}\right)^{(\gamma 2+1 / 2)}+\sum_{i=1}^{n} \varrho_{i} o_{i} c_{i}^{2}+1+\sum_{i=1}^{n} \frac{\mathfrak{J}^{2}}{2} .
\end{aligned}
$$

According to Lemma 5, we obtain

$$
\sum_{i=1}^{n} \sum_{k=1}^{p} \beta_{i}\left(s_{i k}^{2}\right)^{(\gamma 2+1 / 2)}+\sum_{i=1}^{n}\left(\frac{l_{i}}{\kappa_{i}} \widetilde{c}_{i}^{2}\right)^{(1+\gamma 2 / 2)} \geq v_{2}\left(\frac{1}{2^{\gamma 2+12-1}}\right) V_{5}^{(1+\gamma 2 / 2)},
$$

where $\quad v_{2}=\min \left\{\beta_{\min } 2^{(\gamma 2+1 / 2)}, l_{\min }\left(\gamma^{2+1 / 2)} 2^{(\gamma 2+1 / 2)}\right\}\right.$, $\beta_{\text {min }}=\min \left\{\beta_{i}\right\}, l_{\text {min }}=\min \left\{\iota_{i}\right\}$.

From (49), we have

$$
\begin{aligned}
\dot{V}_{1} \leq & -v_{1} V_{1}^{(1+\gamma 1 / 2)}-v_{2}\left(\frac{1}{2^{\gamma 2+12-1}}\right) V_{1}^{(1+\gamma 2 / 2)}-\sum_{i=1}^{n} \frac{\iota_{i}}{\kappa_{i}} \widetilde{c}_{i}^{2} \\
& +\sum_{i=1}^{n}\left(\frac{\iota_{i}}{\kappa_{i}} \widetilde{c}_{i}^{2}\right)^{(\gamma 2+1 / 2)}+\sum_{i=1}^{n} \mathrm{Q}_{i} o_{i} c_{i}^{2}+\sum_{i=1}^{n} \frac{\mathfrak{J}^{2}}{2}+1 .
\end{aligned}
$$

Assume that there exists a compact set $\Pi_{i}$ satisfying

$$
\Pi_{i}=\left\{\left(\tilde{c}_{i}|| \tilde{c}_{i} \mid \leq \Delta_{i}\right\},\right.
$$

where $\Delta_{i}$ is an unknown constant.

If $\Delta_{i}<\sqrt{\left(\zeta_{i} / l_{i}\right)}$, we can obtain

$$
\frac{l_{i}}{\kappa_{i}} \widetilde{c}_{i}^{2}<1,\left(\frac{l_{i}}{\kappa_{i}} \widetilde{c}_{i}^{2}\right)^{(\gamma 2+1 / 2)}<\frac{l_{i}}{\kappa_{i}} \widetilde{c}_{i}^{2} .
$$

If $\Delta_{i} \geq \sqrt{\left(\zeta_{i} / l_{i}\right)}$, we can obtain

$$
\left(\frac{\iota_{i}}{\kappa_{i}} \widetilde{c}_{i}^{2}\right)^{(\gamma 2+1 / 2)}-\frac{l_{i}}{\kappa_{i}} \widetilde{c}_{i}^{2} \leq\left(\frac{l_{i}}{\kappa_{i}} \Delta_{i}^{2}\right)^{(\gamma 2+1 / 2)}-\frac{l_{i}}{\kappa_{i}} \Delta_{i}^{2} .
$$

Denote

$$
v=\sum_{i=1}^{n} \varrho_{i} o_{i} c_{i}^{2}+1+\sum_{i=1}^{n}\left[\left(\frac{\iota_{i}}{\kappa_{i}} \Delta_{i}^{2}\right)^{(\gamma 2+1 / 2)}-\frac{\iota_{i}}{\kappa_{i}} \Delta_{i}^{2}\right] .
$$
that

Then, from the above analyses, we can further conclude

$$
\dot{V}_{1} \leq-v_{1} V_{1}^{(1+\gamma 1 / 2)}-v_{2}\left(\frac{1}{2^{\gamma 2+12-1}}\right)^{n+k-1} V_{1}^{(1+\gamma 2 / 2)}+v .
$$

With Lemma 6, system (6) is practical fixed-time stable. Furthermore, $\mathbf{s}_{i}$ will converge into the region

$$
\Phi=\left\{\lim _{t \rightarrow T_{s}} s_{i} \mid V_{1} \leq \min \left\{v_{1}^{-(2 / 1+\gamma 1)}\left(\frac{v}{1-\theta_{o}}\right)^{(2 / 1+\gamma 1)},\left(v_{2}\left(\frac{1}{2^{v+12-1}}\right)^{n+k-1}\right)^{-(2 / 1+\gamma 1)}\left(\frac{v}{1-\theta_{o}}\right)^{(2 / 1+\gamma 2)}\right\}\right\}
$$


in a fixed-time $T_{s} \leq\left(1 / \vartheta_{1} \theta_{o}(1-\gamma 1+12)\right)+\left(1 / \vartheta_{2}\right.$ $\left.\left(12^{\gamma 2+1} 2-1\right)^{n} \theta_{o}(\gamma 2+12-1)\right)$; that is, $\mathbf{s}_{i}$ will converge into the region $\left\|\mathbf{s}_{i}\right\| \leq \Delta_{s}$ in a fixed-time $T_{s}$.

Theorem 2. When $\mathbf{s}_{i}$ reach the boundary $\Delta_{s}$ in fixed-time, the tracking error $\mathbf{e}_{1 i}$ and $\mathbf{e}_{2 i}$ will converge to

$$
\begin{gathered}
\left|\mathbf{e}_{1 i}\right|<\Delta_{e 1 i}=\max \left\{\varepsilon,\left(\frac{\Delta^{1} k_{s}}{\sigma_{1 i}}\right)^{\left(1 / p_{1}\right)},\left(\frac{\Delta^{1} k_{s}}{\sigma_{2 i}}\right)^{\left(1 / p_{2}\right)}\right\}, \\
\left|\mathbf{e}_{2 i}\right|<\Delta_{e 2 i}=\max \left\{\Delta_{s}+l_{1} \Delta_{e 1 i k}+l_{2} \Delta_{e 1 i k}^{2},\right. \\
\left.\Delta_{s}+\left(\sigma_{1 i} \Delta_{e 1 i}^{p_{1}}+\sigma_{2 i} \Delta_{e 1 i}^{p_{2}}\right)^{k_{1}}\right\},
\end{gathered}
$$

in a fixed time, where $i=1, \ldots, n, k=1,2, \ldots, 6$.

Proof. If $\overline{\mathbf{s}}_{i}=0$, we can obtain

$$
\bar{s}_{i}=\mathbf{e}_{2 i}+\operatorname{sgn}\left(\sigma_{1 i} \operatorname{sgn}\left(\mathbf{e}_{1 i}\right)^{p_{1}}+\sigma_{2 i} \operatorname{sgn}\left(\mathbf{e}_{1 i}\right)^{p_{2}}\right)^{k_{1}}=0, \quad i=1, \ldots, n .
$$

Furthermore, one can obtain

$$
\begin{aligned}
& \dot{\rho}_{e i}+\operatorname{sgn}\left(\sigma_{1 i} \operatorname{sgn}\left(\boldsymbol{\rho}_{e i}\right)^{p_{1}}+\sigma_{2 i} \operatorname{sgn}\left(\boldsymbol{\rho}_{e i}\right)^{p_{2}}\right)^{k_{1}}=0, \quad i=1, \ldots, n, \\
& \boldsymbol{\omega}_{e i}+\operatorname{sgn}\left(\sigma_{1 i} \operatorname{sgn}\left(\mathbf{q}_{e i}\right)^{p_{1}}+\sigma_{2 i} \operatorname{sgn}\left(\mathbf{q}_{e i}\right)^{p_{2}}\right)^{k_{1}}=0, \quad i=1, \ldots, n .
\end{aligned}
$$

Construct the following Lyapunov function candidate:

$$
\begin{aligned}
V_{4} & =\frac{1}{2} \boldsymbol{\rho}_{e i}^{T} \boldsymbol{\rho}_{e i}+\mathbf{q}_{e i}^{T} \mathbf{q}_{e i}+\left(1-q_{0 e i}\right)^{2}, \\
& =\frac{1}{2} \boldsymbol{\rho}_{e i}^{T} \boldsymbol{\rho}_{e i}+2\left(1-q_{0 e i}\right) .
\end{aligned}
$$

Denote

$$
V_{5}=\boldsymbol{\rho}_{e i}^{T} \boldsymbol{\rho}_{e i}, V_{6}=2\left(1-q_{0 e i}\right) .
$$

Taking the derivative of $V_{5}$ and $V_{6}$ yields

$$
\begin{aligned}
\dot{V}_{5} & =\boldsymbol{\rho}_{e i}^{T} \dot{\rho}_{e i}, \\
& =\boldsymbol{\rho}_{e i}^{T}\left(-\operatorname{sgn}\left(\sigma_{1 i} \operatorname{sgn}\left(\boldsymbol{\rho}_{e i}\right)^{p_{1}}+\sigma_{2 i} \operatorname{sgn}\left(\boldsymbol{\rho}_{e i}\right)^{p_{2}}\right)^{k_{1}}\right), \\
& -\left|\sigma_{1 i}\left(\rho_{e i}^{T} \rho_{e i}\right)^{\left(\left(1 / k_{1}\right)+p_{1} / 2\right)}+\sigma_{2 i}\left(\rho_{e i}^{T} \rho_{e i}\right)^{\left(\left(1 / k_{1}\right)+p_{21} / 2\right)}\right|^{k_{1}}, \\
& \leq-\left(\sigma_{1 i}{ }^{\left(\left(1 / k_{1}\right)+p_{1} / 2\right)} V_{5}^{\left(\left(1 / k_{1}\right)+p_{1} / 2\right)}+\sigma_{2 i} 2^{\left(\left(1 / k_{1}\right)+p_{2} / 2\right)} V_{5}^{\left(\left(1 / k_{1}\right)+p_{2} / 2\right)}\right)^{k_{1}} .
\end{aligned}
$$

Similarly, taking the derivative of $V_{6}$ yields

$$
\begin{aligned}
& \dot{V}_{6}=-2 \dot{q}_{0 e i}, \\
& =\mathbf{q}_{i e v}^{T} \boldsymbol{\omega}_{e i}, \\
& =-\mathbf{q}_{i e v}^{T}\left(\operatorname{sgn}\left(\sigma_{1 i} \operatorname{sgn}\left(\mathbf{q}_{e i}\right)^{p_{1}}+\sigma_{2 i} \operatorname{sgn}\left(\mathbf{q}_{e i}\right)^{p_{2}}\right)^{k_{1}}\right), \\
& =-\left|\sigma_{1 i}\left(\mathbf{q}_{i e v}^{T} \mathbf{q}_{i e v}\right)^{\left(\left(1 / k_{1}\right)+p_{1} / 2\right)}+\sigma_{2 i}\left(\mathbf{q}_{i e v}^{T} \mathbf{q}_{i e v}\right)\left(\left(1 / k_{1}\right)+p_{2} / 2\right)\right|^{k_{1}}, \\
& \leq-\left(\sigma_{1 i} 0.5^{\left(\left(1 / k_{1}\right)+p_{1} / 2\right)} V_{6}^{\left(\left(1 / k_{1}\right)+p_{1} / 2\right)}+\sigma_{2 i} 0.5^{\left(\left(1 / k_{1}\right)+p_{2} / 2\right)} V_{6}^{\left(\left(1 / k_{1}\right)+p_{2} / 2\right)}\right)^{k_{1}} \text {. }
\end{aligned}
$$

Then, from (62) and (63), we can obtain that system error states $\left(\rho_{e i}, \mathbf{q}_{e i}\right)$ converge into regions $(0,0)$, at the same time $\dot{q}_{0 e i} \in(-1,1]$ converges to 1 in fixed-time by using Lemma 6.
If $\overline{\mathbf{s}}_{i} \neq 0$ and $\left|e_{1 i k}\right|<\varepsilon$, which implies that $\left|e_{1 i k}\right|$ has converged to the region $\left|e_{1 i k}\right|<\Delta_{e 1 i k}=\varepsilon$ in a fixed time. Then, from (23), we have 


$$
e_{2 i k}+l_{1 i} e_{1 i k}+l_{2 i} \operatorname{sgn}\left(e_{1 i k}\right)^{2}=\omega_{i k},\left|\varpi_{i k}\right| \leq \Delta_{s} .
$$

Moreover, it is easy to see that

$$
\left|e_{2 i k}\right|<\Delta_{s}+l_{1 i} \Delta_{e 1 i k}+l_{2 i} \Delta_{e 1 i k}^{2}
$$

which means that $\left|e_{2 i k}\right|$ converges to the region $\left|e_{1 i k}\right|<\Delta_{e l i k}=\varepsilon$ in a fixed time.

If $\bar{s}_{i} \neq 0$ and $\left|e_{1 i k}\right|>\varepsilon$, we can obtain

$$
\bar{s}_{i}=\mathbf{e}_{2 i}+\operatorname{sgn}\left(\sigma_{1 i} \operatorname{sgn}\left(\mathbf{e}_{1 i}\right)^{p_{1}}+\sigma_{2 i} \operatorname{sgn}\left(\mathbf{e}_{1 i}\right)^{p_{2}}\right)^{k_{1}} \neq 0, \quad i=1, \ldots, n,
$$

which means

$$
\bar{s}_{i}=\mathbf{e}_{2 i}+\operatorname{sgn}\left(\sigma_{1 i} \operatorname{sgn}\left(\mathbf{e}_{1 i}\right)^{p_{1}}+\sigma_{2 i} \operatorname{sgn}\left(\mathbf{e}_{1 i}\right)^{p_{2}}\right)^{k_{1}}=\varliminf_{i k},\left|\varpi_{i k}\right| \leq \Delta_{s} .
$$

After a simple transformation, (67) can be rewritten as

$$
\mathbf{e}_{2 i}+\left(1-\frac{\omega_{i k}}{\operatorname{sgn}\left(\sigma_{1 i} \operatorname{sgn}\left(\mathbf{e}_{1 i}\right)^{\gamma 1}+\sigma_{2 i} \operatorname{sgn}\left(\mathbf{e}_{1 i}\right)^{\gamma 2}\right)^{k_{1}}}\right) \operatorname{sgn}\left(\sigma_{1 i} \operatorname{sgn}\left(\mathbf{e}_{1 i}\right)^{\gamma 1}+\sigma_{2 i} \operatorname{sgn}\left(\mathbf{e}_{1 i}\right)^{\gamma 2}\right)^{k_{1}}=0 .
$$

When $1-\left(\oint_{i k} / \operatorname{sgn}\left(\sigma_{1 i} \operatorname{sgn}\left(\mathbf{e}_{1 i}\right)^{p_{1}}+\sigma_{2 i} \operatorname{sgn}\left(\mathbf{e}_{1 i}\right)^{p_{2}}\right)^{k_{1}}\right)>0$, (66) is still kept in the form of the FTTSM as in Case 1, which implies that

$$
\left|\mathbf{e}_{1 i}\right|<\left(\frac{\Delta^{1} k_{s}}{\sigma_{1 i}}\right)^{\left(1 / p_{1}\right)},\left|\mathbf{e}_{1 i}\right|<\left(\frac{\Delta^{1} k_{s}}{\sigma_{2 i}}\right)^{\left(1 / p_{2}\right)} .
$$

Thus, system state $\mathbf{e}_{1 i}$ will converge into the region

$$
\left|\mathbf{e}_{1 i}\right|<\Delta_{e 1 i}=\max \left\{\left(\frac{\Delta^{1} k_{s}}{\sigma_{1 i}}\right)^{\left(1 / p_{1}\right)},\left(\frac{\Delta^{1} k_{s}}{\sigma_{2 i}}\right)^{\left(1 / p_{2}\right)}\right\} \text {, }
$$

in a fixed time.

On the other hand, from (67), we can obtain that system state $\mathbf{e}_{2 i}$ will converge to the region

$$
\left|e_{2 i}\right|<\Delta_{e 2 i}=\Delta_{s}+\left(\sigma_{1 i} \Delta_{e 1 i}^{p_{1}}+\sigma_{2 i} \Delta_{e 1 i}^{p_{2}}\right)^{k_{1}},
$$

in a fixed time.

Furthermore, we can conclude that

$$
\begin{gathered}
\left|e_{1 i}\right|<\Delta_{e 1 i}=\max \left\{\varepsilon,\left(\frac{\Delta^{1} k_{s}}{\sigma_{1 i}}\right)^{\left(1 / p_{1}\right)},\left(\frac{\Delta^{1} k_{s}}{\sigma_{2 i}}\right)^{\left(1 / p_{2}\right)}\right\}, \\
\left|e_{2 i}\right|<\Delta_{e 2 i}=\max \left\{\Delta_{s}+l_{1} \Delta_{e 1 i k}+l_{2} \Delta_{e 1 i k}^{2},\right. \\
\left.\Delta_{s}+\left(\sigma_{1 i} \Delta_{e 1 i}^{p_{1}}+\sigma_{2 i} \Delta_{e 1 i}^{p_{2}}\right)^{k_{1}}\right\} .
\end{gathered}
$$

Thus, $\mathbf{e}_{1 i}$ and $\mathbf{e}_{2 i}, i=1,2,3$, will converge to the regions $\Delta_{e 1 i}$ and $\Delta_{e 2 i}$ in a fixed time, respectively.

Remark 4. Based on the multispacecraft FTTSM results, the property of graph theory and adaptive technique, a fixedtime 6-DOF coordinated control strategy is designed under directed communication topology. Subsequently, the system tracking error states can be guaranteed to converge their desired trajectories in a fixed time even with external disturbances and quantized control input. Note that this small region is determined by the controller parameters $\alpha_{i}>0$, $\beta_{i}>0$, and $\kappa_{i}>0$. Thus, this small region is adjustable and can be reduced as needed.

Remark 5. By employing the adaptive method, the precise information of the external disturbance and parameters uncertain is not needed for the controller (28) design. Moreover, it is no required to make an additional assumption about interspacecraft communication topology in the designed fixed-time coordinated controller. Hence, the designed controller is suitable for any communication topology. Even if there is no communication link between the formation spacecraft, this proposed controller can still guarantee the practical fixed-time stability of each formation spacecraft.

\section{Illustrative Example}

To validate the proposed coordinated controller, we give an illustrative example in this section. The communicate topology of three follower spacecraft is described in Figure 2, in which "Sat $i(i=1,2,3)$ " denotes the $i$ th formation spacecraft. The leader spacecraft is specified to a circular orbit with a radius of $6878 \mathrm{~km}$ and orbit angel velocity is $n_{0}=1.11 \times 10^{-3} \mathrm{rad} / \mathrm{s}$.

The weighted Laplace matrix $\mathbf{L}$ is designed as

$$
\mathbf{L}=\left[\begin{array}{ccc}
1 & 0 & -1 \\
-1 & 1 & 0 \\
0 & -1 & 1
\end{array}\right]
$$

The inertia matrix and mass are

$$
\begin{aligned}
\mathbf{J}_{1} & =\left[\begin{array}{ccc}
10 & 1 & 0.4 \\
1 & 8 & 0.2 \\
0.4 & 0.2 & 7
\end{array}\right] \mathrm{kg} \cdot \mathrm{m}^{2}, \mathbf{J}_{2}=\left[\begin{array}{ccc}
11 & 0.5 & 0.4 \\
0.5 & 9 & 0.2 \\
0.4 & 0.2 & 7
\end{array}\right] \mathrm{kg} \cdot \mathrm{m}^{2}, \\
\mathbf{J}_{3} & =\left[\begin{array}{ccc}
9 & 0.5 & 0.7 \\
0.5 & 3.5 & 0.3 \\
0.7 & 0.2 & 8
\end{array}\right] \mathrm{kg} \cdot \mathrm{m}^{2}, m_{f i}=50 \mathrm{~kg}, \quad \forall i=1,2,3 .
\end{aligned}
$$




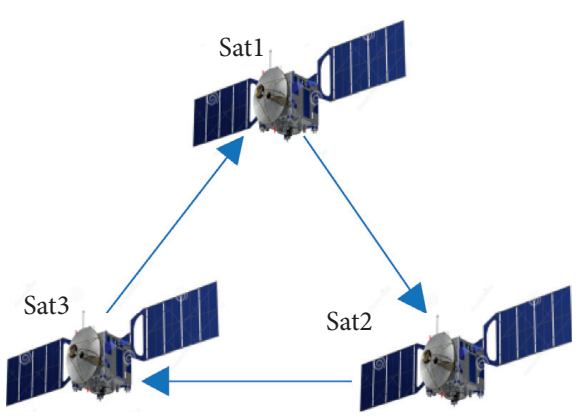

Figure 2: The directed communication topology of three follower spacecraft.
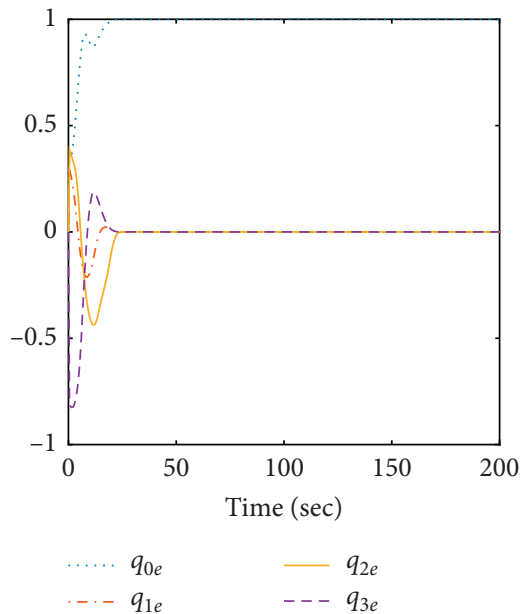

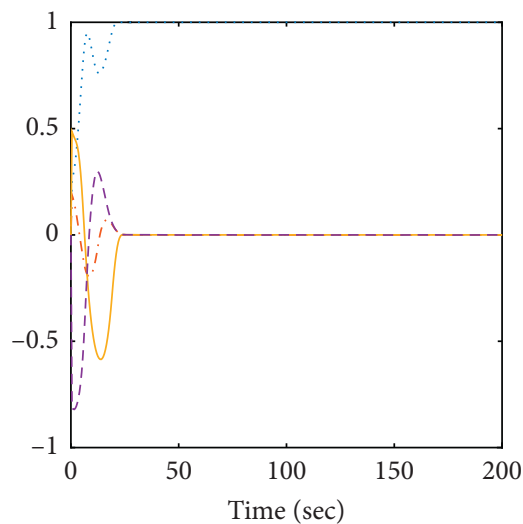

$q_{2 e}$ $---q_{3 e}$

(b)
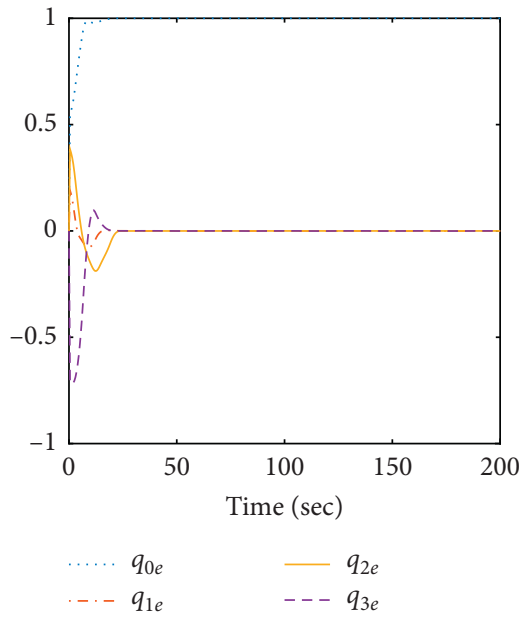

(c)

Figure 3: The curves of the relative attitude error. (a) Sat1. (b) Sat2. (c) Sat3.

The desired attitude quaternion and attitude angular velocity are

$$
\mathbf{q}_{d i}=\left[\begin{array}{llll}
1 & 0 & 0 & 0
\end{array}\right]^{T}, \boldsymbol{\omega}_{d i}=\left[\begin{array}{lll}
0 & 0 & 0
\end{array}\right]^{T} \mathrm{rad} / \mathrm{s}, \quad \forall i=1,2,3 .
$$

$$
\begin{aligned}
& \mathbf{q}_{1}(0)=\left[\begin{array}{llll}
0.3317 & 0.3 & 0.4 & -0.8
\end{array}\right]^{T}, \boldsymbol{\omega}_{1}(0)=\left[\begin{array}{lll}
0.07 & -0.05 & -0.04
\end{array}\right]^{T} \mathrm{rad} / \mathrm{s}, \\
& \mathbf{q}_{2}(0)=\left[\begin{array}{llll}
0.2646 & 0.2 & 0.5 & -0.8
\end{array}\right]^{T}, \boldsymbol{\omega}_{2}(0)=\left[\begin{array}{lll}
0.07 & -0.05 & -0.04
\end{array}\right]^{T} \mathrm{rad} / \mathrm{s}, \\
& \mathbf{q}_{3}(0)=\left[\begin{array}{llll}
0.5568 & 0.2 & 0.4 & -0.7
\end{array}\right]^{T}, \boldsymbol{\omega}_{3}(0)=\left[\begin{array}{lll}
0.06 & -0.05 & -0.05
\end{array}\right]^{T} \mathrm{rad} / \mathrm{s} .
\end{aligned}
$$

The relative position and velocity are initialized: $\boldsymbol{\rho}_{i}(0)=\left[\begin{array}{lll}-10 & 160 & 50\end{array}\right]^{T} \mathrm{~m}, v_{i}(0)=\left[\begin{array}{lll}0 & 0 & 0\end{array}\right]^{T} \mathrm{~m} / \mathrm{s}, \quad \forall i=1,2,3$.

In order to form a triangle of three follower spacecraft, the desired relative position and velocity are specified as

$$
\begin{aligned}
& \boldsymbol{\rho}_{1 d}=\left[\begin{array}{lll}
0 & 100 & 0
\end{array}\right]^{T} \mathrm{~m}, \boldsymbol{\rho}_{2 d}=\left[\begin{array}{lll}
0 & 200 & 0
\end{array}\right]^{T} \mathrm{~m}, \\
& \boldsymbol{\rho}_{3 d}=\left[\begin{array}{lll}
0 & 150 & 50 \sqrt{3}
\end{array}\right]^{T} \mathrm{~m}, \mathbf{v}_{d i}(0)=\left[\begin{array}{lll}
0 & 0 & 0
\end{array}\right]^{T} \mathrm{~m} / \mathrm{s}, \quad \forall i=1,2,3 .
\end{aligned}
$$

The external disturbance of torque and force are specified as 


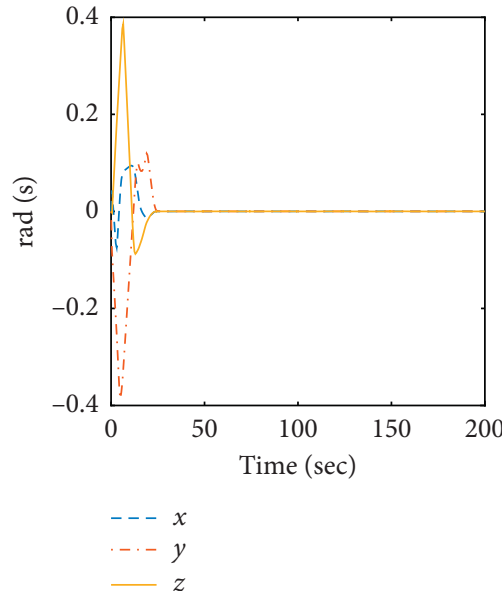

(a)

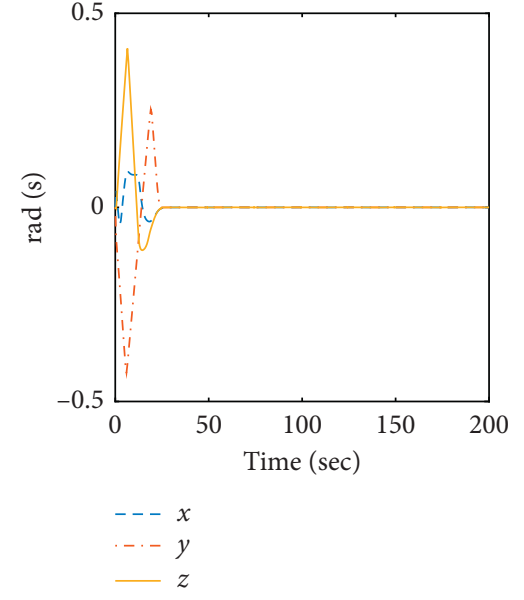

(b)

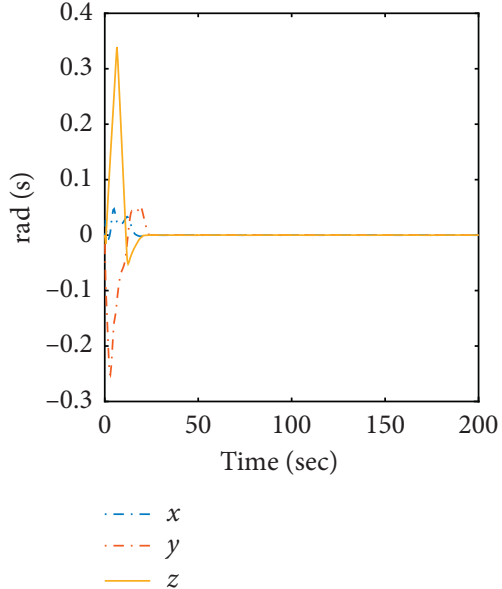

(c)

FIgURE 4: The curves of the relative angular velocity error. (a) Sat1. (b) Sat2. (c) Sat3.

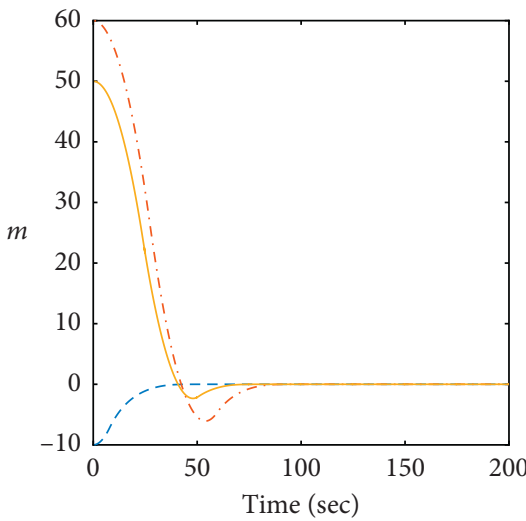

$--x$
$--y$
$-z$
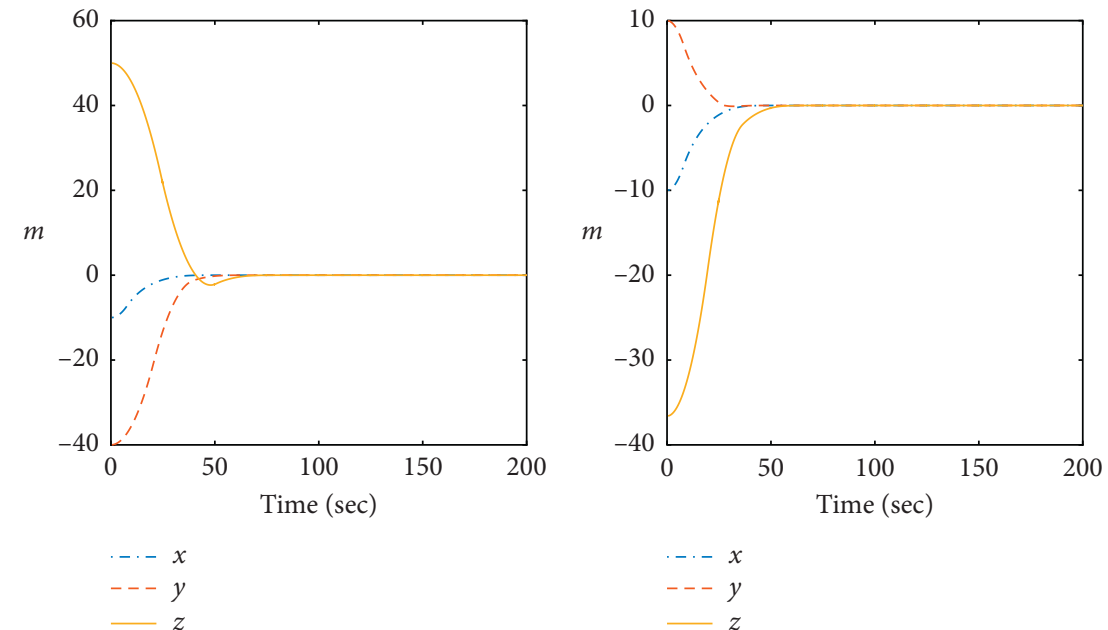

(a)

$$
\begin{array}{r}
-x \\
--y \\
--y
\end{array}
$$

(c)

Figure 5: The curves of the relative position error. (a) Sat1. (b) Sat2. (c) Sat3.
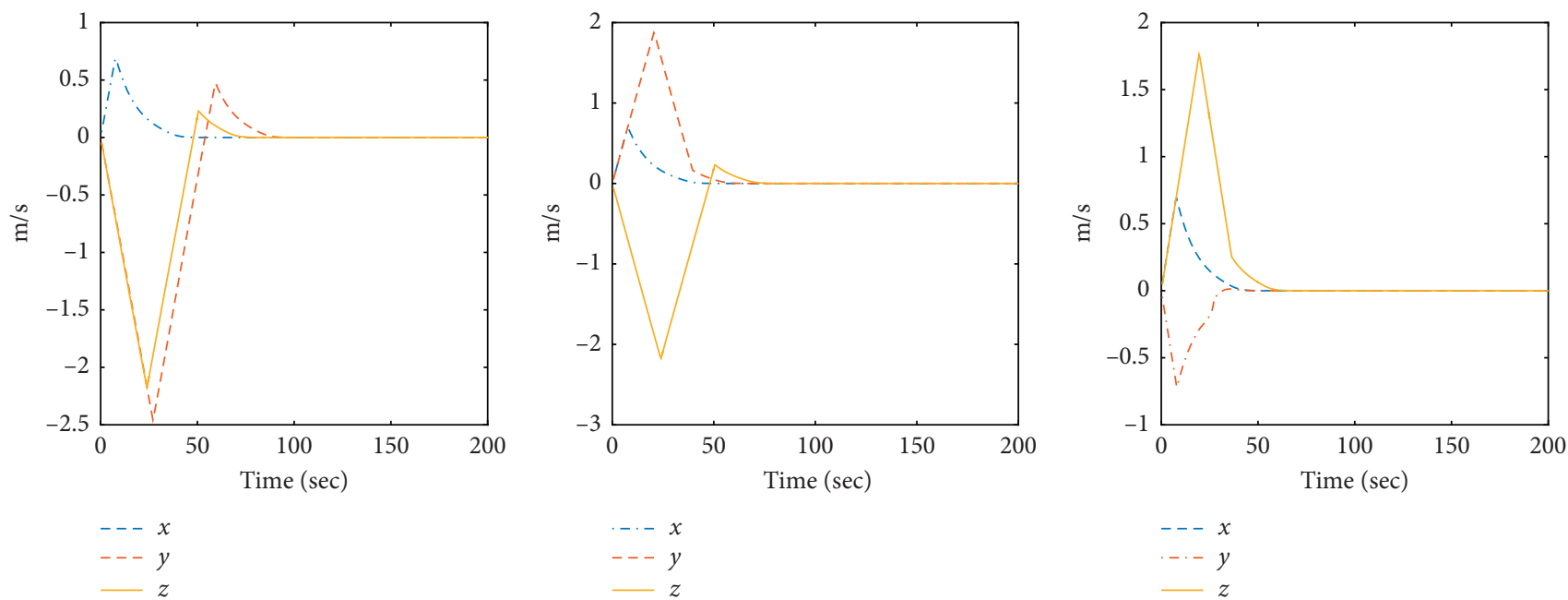

(a)

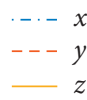

(c)

Figure 6: The curves of the relative velocity error. (a) Sat1. (b) Sat2. (c) Sat3. 


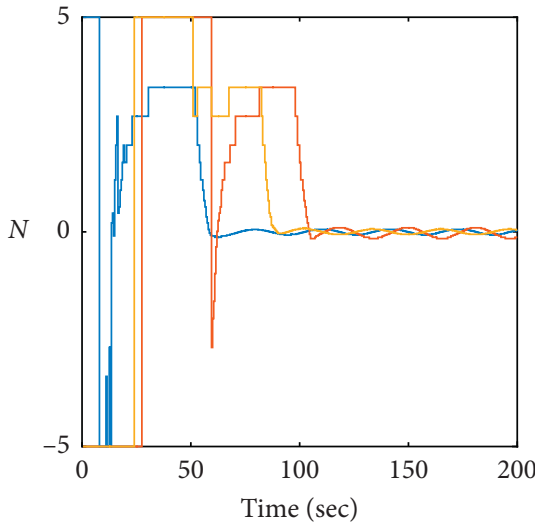

$x$
$-y$
$-z$
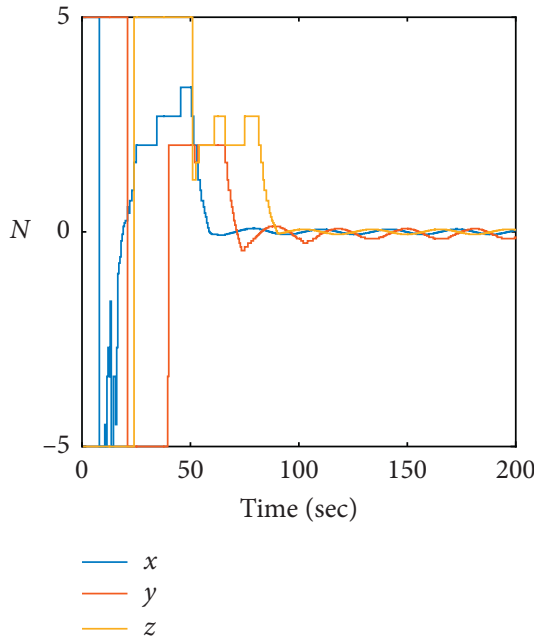

(a)

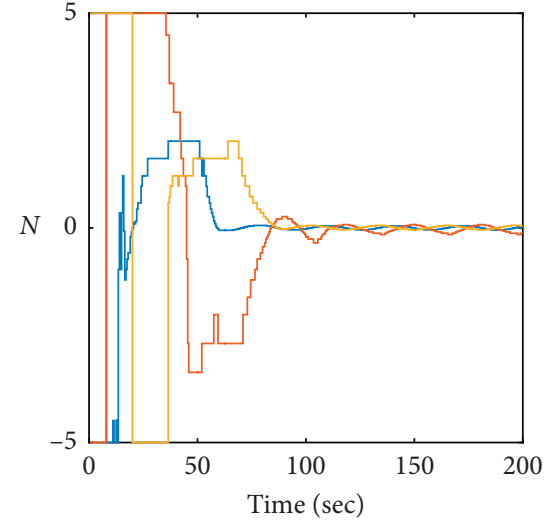

$x$
$-y$
$-\quad z$ (c)

Figure 7: The curves of the quantized control force. (a) Sat1. (b) Sat2. (c) Sat3.

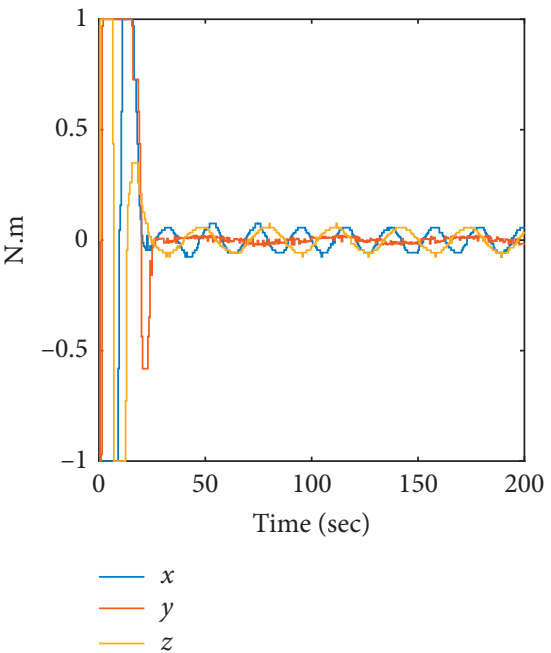

(a)
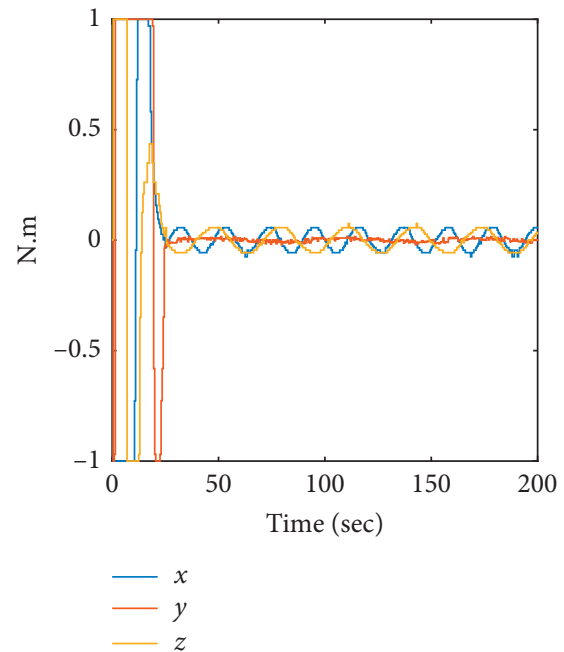

(b)

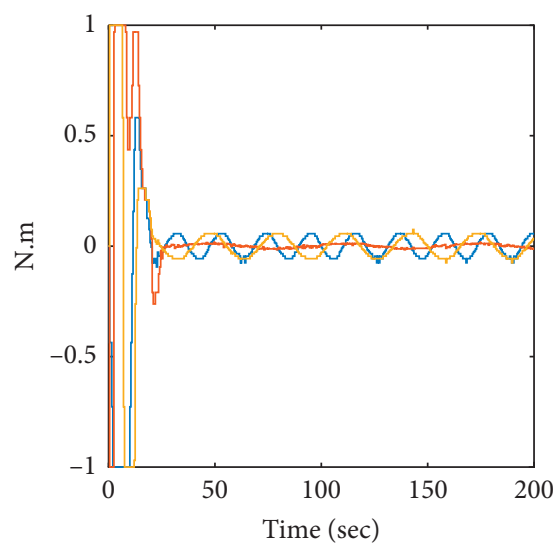

Figure 8: The curves of the quantized control torque. (a) Sat1. (b) Sat2. (c) Sat3.
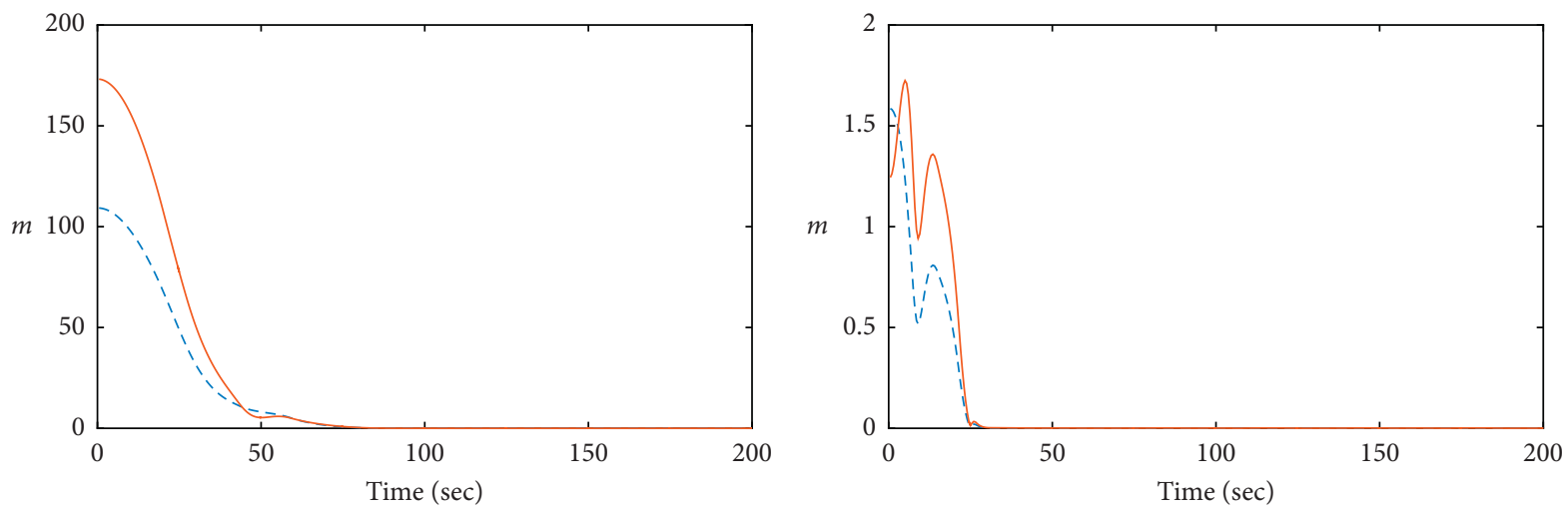

$---\bar{\omega} 1$

$---\bar{\omega} 3$

$-\bar{\omega} 4$

(a)

(b)

Figure 9: The curves of the performance indexes $₫ 1$ to $₫ 4$. 


$$
\begin{aligned}
\mathbf{z}_{i} & =\left[\begin{array}{lll}
0.06 \cos (0.3 t) & 0.01 \sin (0.1 t) & 0.06 \cos (0.2 t)
\end{array}\right]^{T} \mathrm{Nm}, \\
\mathbf{F}_{i t} & =\left[\begin{array}{ll}
0.001 \cos (0.02 t) & 0.002 \sin (0.02 t)-0.001 \sin (0.02 t)
\end{array}\right]^{T} \mathrm{~N}, \quad \forall i=1,2,3 .
\end{aligned}
$$

The maximum of input control force and torques are limited to $5 \mathrm{~N}$ and $1 \mathrm{Nm}$, respectively.

The following performance indexes are defined to describe the synchronization accuracy and tracking accuracy of the proposed control law (28):

$$
\begin{aligned}
& \bowtie 1=\sqrt{\sum_{i=1}^{3}\left\|\tilde{\rho}_{i}\right\|^{2}}, \omega 2=\sqrt{\sum_{i=1}^{3} \sum_{j \in N_{i}}\left\|\tilde{\rho}_{i, j}\right\|^{2}}, \\
& ₫ 3=\sqrt{\sum_{i=1}^{3} \mathbf{q}_{v e i}^{T} \mathbf{q}_{v e i}, \Phi 4}=\sqrt{\sum_{i=1}^{3} \sum_{j \in N_{i}} \mathbf{q}_{i, j v e}^{T} \mathbf{q}_{i, j v e},}
\end{aligned}
$$

where $\tilde{\rho}_{i}=\rho_{i}-\rho_{d i}, \tilde{\rho}_{j}=\rho_{j}-\rho_{j d}, \widetilde{\rho}_{i, j}=\widetilde{\rho}_{i}-\tilde{\rho}_{j}$, and $\mathbf{q}_{i, j v e}$ is the vector part of $\mathbf{q}_{i, j e}=\left[\begin{array}{ll}q_{i, j 0 e} & \mathbf{q}_{i, j v e}^{T}\end{array}\right]^{T}=\mathbf{q}_{e i} \otimes \mathbf{q}_{e j}$. From the definition of $₫ 1, \bowtie 2, \bowtie 3$, and $₫ 4$, it can be shown that smaller $₫ 1$ and $₫ 3$ can guarantee the better formation and attitude tracking performance, respectively; smaller $₫ 2$ and $₫ 4$ can ensure the better formation and attitude synchronization performance, respectively.

The control scheme (28), adaptive updating law (29), and hysteretic quantizer (12) parameters are chosen as $\gamma 1=0.9$, $\gamma 2=1.1, a_{12}=0.5, a_{23}=0.5, a_{31}=0.5, \alpha_{i}=0.1, \quad \beta_{i}=1$, $p_{1}=0.4, p_{2}=1.5, k_{1}=2, c_{i}=0.1, b_{i}=0.04, \kappa_{i}=0.000002$, $\Im_{i}=0.5, \quad \varrho_{i}=0.000002, \forall i=1,2,3, \quad \delta=0.25, \quad$ and $u_{\text {min }}=0.00001$.

The simulation results of the controller (28) are shown in Figures 3 to 9, where Figure 3 depicts the relative attitude error and Figure 4 plots the relative angular velocity error. It can be observed that the relative attitude errors converge to near zero within $50 \mathrm{~s}$, which has a fast convergence rate. The relative position error and relative velocity error are shown in Figures 5 and 6, respectively. It can be seen that the relative position errors converge to near zero about $85 \mathrm{~s}$, which has a fast convergence rate. The quantized control force and torque are shown in Figures 7 and 8 , respectively. The curves of the performance indexes $₫ 1$ to $₫ 4$ are depicted in Figure 9. As seen from the simulation results, the proposed fixed-time control strategy provides a good performance of tracking and synchronization.

\section{Conclusion}

In this paper, the fixed-time 6-DOF coordinated control problem has been studied for multiple spacecraft formation with input quantization under directed communication graph. A fixed-time adaptive coordinated control strategy is designed by using multispacecraft FTTSM vector such that, in the presence of the upper bounds of unknown external disturbances, the controlled system is practical fixed-time stable and, at the same time, the tracking errors converge to their desired trajectories in a fixed time. Compared with the existing finite-time stabilization controllers, the designed adaptive fixed-time coordinated controller in this paper is more suitable for practical engineering application due to its convergent time regardless of initial system states. An illustrative example is given to illustrate the performance of the presented fixed-time coordinated controller. It was shown that the presented controller not only can ensure each spacecraft's convergence to its desired states but also can provide the desired synchronization and tacking performance. Future study will focus on the extension of the presented controller under time-varying communication topology and communication time delay.

\section{Data Availability}

The data used to support the findings of this study are included within the article.

\section{Conflicts of Interest}

The authors declare that they have no conflicts of interest.

\section{Acknowledgments}

The authors of this paper acknowledge the financial support of the National Natural Science Foundation of China (nos. 11902255, U1837601, and U1233202).

\section{References}

[1] X. Cao, P. Shi, Z. Li, and M. Liu, "Neural-network-based adaptive backstepping control with application to spacecraft attitude regulation," IEEE Transactions on Neural Networks and Learning Systems, vol. 29, no. 9, pp. 4303-4313, 2018.

[2] D. Lee, A. K. Sanyal, and E. A. Butcher, "Asymptotic tracking control for spacecraft formation flying with decentralized collision avoidance," Journal of Guidance, Control, and Dynamics, vol. 38, no. 4, pp. 587-600, 2015.

[3] Q. Liu, M. Liu, and J. Yu, "Adaptive fault-tolerant control for attitude tracking of flexible spacecraft with limited data transmission," IEEE Transactions on Systems, Man, and Cybernetics: Systems, p. 1, 2019.

[4] D. Ye, M. Shi, and Z. Sun, "Satellite proximate interception vector guidance based on differential games," Chinese Journal of Aeronautics, vol. 31, no. 6, pp. 1352-1361, 2018.

[5] D. Ye, M. Shi, and Z. Sun, "Satellite proximate pursuit-evasion game with different thrust configurations," Aerospace Science and Technology, vol. 99, Article ID 105715, 2020.

[6] G. Dong, L. Cao, D. Yao, H. Li, and R. Lu, "Adaptive attitude control for multi-MUAVs with output dead-zone and actuator fault," IEEE/CAA Journal of Automatica Sinica, 2020.

[7] C. Yue, K. D. Kumar, Q. Shen, C. H. Goh, and T. H. Lee, "Attitude stabilization using two parallel single-gimbal control moment gyroscopes," Journal of Guidance, Control, and Dynamics, vol. 42, no. 2, pp. 1353-1364, 2019. 
[8] Y. Lv, Q. Hu, G. Ma, and J. Zhou, “6 DOF synchronized control for spacecraft formation flying with input constraint and parameter uncertainties," ISA Transactions, vol. 50, no. 4, pp. 573-580, 2011.

[9] L. Sun and W. Huo, "6-DOF integrated adaptive backstepping control for spacecraft proximity operations," IEEE Transactions on Aerospace and Electronic Systems, vol. 51, no. 3, pp. 2433-2443, 2015.

[10] C. Liu, K. Shi, X. Yue, and Z. Sun, "Inertia-free saturated output feedback attitude stabilization for uncertain spacecraft," International Journal of Robust and Nonlinear Control, vol. 30, no. 13, pp. 5101-5121, 2020.

[11] C. Liu, X. Yue, K. Shi, and Z. Sun, "Inertia-free attitude stabilization for flexible spacecraft with active vibration suppression," International Journal of Robust and Nonlinear Control, vol. 29, no. 18, pp. 6311-6336, 2019.

[12] J. Na, Y. Huang, X. Wu, G. Gao, G. Herrmann, and J. Z. Jiang, "Active adaptive estimation and control for vehicle suspensions with prescribed performance," IEEE Transactions on Control Systems Technology, vol. 26, no. 6, pp. 2063-2077, 2018.

[13] Z. Zhu, Y. Pan, Q. Zhou, and C. Lu, "Event-triggered adaptive fuzzy control for stochastic nonlinear systems with unmeasured states and unknown backlash-like hysteresis," IEEE Transactions on Fuzzy Systems, p. 1, 2020.

[14] T. Chen, J. Shan, and H. Wen, "Distributed passivity-based control for multiple flexible spacecraft with attitude-only measurements," Aerospace Science and Technology, vol. 94, Article ID 105408, 2019.

[15] P. Li, X. Yu, and Y. Zhang, "The design of quasi-optimal higher order sliding mode control via disturbance observer and switching-gain adaptation," IEEE Transactions on Systems, Man, and Cybernetics: Systems, vol. 50, no. 11, pp. 4817-4827, 2020.

[16] Q. Li and Z. Deng, "Coordinated orbit-attitude-vibration control of a sun-facing solar power satellite," Journal of Guidance, Control, and Dynamics, vol. 42, no. 8, pp. 18631869, 2019.

[17] Z. Ning, Y. Xia, K. Lu, and Y. Li, "Decentralised finite-time attitude synchronisation and tracking control for rigid spacecraft," International Journal of Systems Science, vol. 46, no. 14, pp. 2493-2509, 2015.

[18] Q. Zhou, S. Zhao, H. Li, R. Lu, and C. Wu, "Adaptive neural network tracking control for robotic manipulators with dead zone," IEEE Transactions on Neural Networks and Learning Systems, vol. 30, no. 12, pp. 3611-3620, 2019.

[19] P. Du, Y. Pan, H. Li, and H.-K. Lam, "Nonsingular finite-time event-triggered fuzzy control for large-scale nonlinear systems," IEEE Transactions on Fuzzy Systems, p. 1, 2020.

[20] G. Lin, H. Li, H. Ma, D. Yao, and R. Lu, "Human-in-the-loop consensus control for nonlinear multi-agent systems with actuator faults," IEEE/CAA Journal of Automatica Sinica, 2020.

[21] M. Liu, L. Zhang, and W. X. Zheng, "Fault reconstruction for stochastic hybrid systems with adaptive discontinuous observer and non-homogeneous differentiator," Automatica, vol. 85, pp. 339-348, 2017.

[22] J. Na, Y. Huang, X. Wu, S.-F. Su, and G. Li, “Adaptive finitetime fuzzy control of nonlinear active suspension systems with input delay," IEEE Transactions on Cybernetics, vol. 50, no. 6, pp. 2639-2650, 2020.

[23] B. Huang, A.-J. Li, Y. Guo, and C.-Q. Wang, "Rotation matrix based finite-time attitude synchronization control for spacecraft with external disturbances," ISA Transactions, vol. 85, pp. 141-150, 2019.

[24] J. Na, S. Wang, Y. Liu, Y. Huang, and X. Ren, "Finite-time convergence adaptive neural network control for nonlinear servo systems," IEEE Transactions on Cybernetics, vol. 50, no. 6, pp. 2568-2579, 2019.

[25] H. Zhang, D. Yue, X. Yin, S. Hu, and C. X. Dou, "Finite-time distributed event-triggered consensus control for multi-agent systems," Information Sciences, vol. 339, pp. 132-142, 2016.

[26] Y. Xia, J. Zhang, K. Lu, and N. Zhou, Finite Time and Cooperative Control of Flight Vehicles, Springer, Singapore, 2019.

[27] A. Polyakov, "Nonlinear feedback design for fixed-time stabilization of linear control systems," IEEE Transactions on Automatic Control, vol. 57, no. 8, pp. 2106-2110, 2012.

[28] Y. Wu and Z. Wang, "Fuzzy adaptive practical fixed-time consensus for second-order nonlinear multiagent systems under actuator faults," IEEE Transactions on Cybernetics, p. 1, 2020.

[29] X. Yu, P. Li, and Y. Zhang, "Fixed-time actuator fault accommodation applied to hypersonic gliding vehicles," IEEE Transactions on Automation Science and Engineering, p. 1, 2020.

[30] A. Polyakov, "Distributed fixed-time attitude coordination control for multiple rigid spacecraft," International Journal of Robust and Nonlinear Control, vol. 2019, pp. 1-16, 2019.

[31] Z. Zuo, "Nonsingular fixed-time consensus tracking for second-order multi-agent networks," Automatica, vol. 54, pp. 305-309, 2015.

[32] H. Liang, X. Guo, Y. Pan, and T. Huang, "Event-Triggered fuzzy bipartite tracking control for network systems based on distributed reduced-order observers (revised manuscript of TFS-2019-1049)," IEEE Transactions on Fuzzy Systems, p. 1, 2020.

[33] M. Liu, L. Zhang, P. Shi, and Y. Zhao, "Fault estimation sliding-mode observer with digital communication constraints," IEEE Transactions on Automatic Control, vol. 63, no. 10, pp. 3434-3441, 2018.

[34] Z. Zuo, X. Cao, and Y. Wang, "Security control of multi-agent systems under false data injection attacks," Neurocomputing, vol. 404, pp. 240-246, 2020.

[35] Z. Zuo, P. Xie, and Y. Wang, "Output-based dynamic eventtriggering control for sensor saturated systems with external disturbance," Applied Mathematics and Computation, vol. 374, Article ID 125043, 2020.

[36] A. Liu, W.-A. Zhang, L. Yu, H. Yan, and R. Zhang, "Formation control of multiple mobile robots incorporating an extended state observer and distributed model predictive approach," IEEE Transactions on Systems, Man, and Cybernetics: Systems, vol. 50, no. 11, pp. 4587-4597, 2020.

[37] C. Liu, G. Vukovich, Z. Sun, and K. Shi, "Observer-based fault-tolerant attitude control for spacecraft with input delay," Journal of Guidance, Control, and Dynamics, vol. 41, no. 9, pp. 2041-2053, 2018.

[38] M. Liu, L. Zhang, P. Shi, and Y. Zhao, "Sliding mode control of continuous-time Markovian jump systems with digital data transmission," Automatica, vol. 80, pp. 200-209, 2017.

[39] D. Wang, X. Mei, R. Weng, Z. Qu, and L. Zhang, "Hybrid filter design of fault detection for networked linear systems with variable packet dropout rate," IET Control Theory and Applications, vol. 13, no. 9, pp. 1239-1245, 2019.

[40] H. Li, P. Shi, D. Yao, and L. Wu, "Observer-based adaptive sliding mode control for nonlinear Markovian jump systems," Automatica, vol. 64, pp. 133-142, 2016. 
[41] A. Liu, W.-A. Zhang, M. Z. Q. Chen, and L. Yu, "Moving horizon estimation for mobile robots with multirate sampling," IEEE Transactions on Industrial Electronics, vol. 64, no. 2, pp. 1457-1467, 2017.

[42] R. Liu, X. Cao, M. Liu, and Y. Zhu, “6-DOF fixed-time adaptive tracking control for spacecraft formation flying with input quantization," Information Sciences, vol. 475, pp. 82-99, 2019.

[43] H. Ma, H. Li, R. Lu, and T. Huang, "Adaptive event-triggered control for a class of nonlinear systems with periodic disturbances," Science China Information Sciences, vol. 63, no. 5, Article ID 150212, 2020.

[44] R. Merris, "Laplacian matrices of graphs: a survey," Linear Algebra and Its Applications, vol. 197, no. 198, pp. 143-176, 1994.

[45] F. Wang, Z. Liu, Y. Zhang, and C. L. P. Chen, "Adaptive quantized controller design via backstepping and stochastic small-gain approach," IEEE Transactions on Fuzzy Systems, vol. 24, no. 2, pp. 330-343, 2016.

[46] B. Wu, D. Wang, and E. K. Poh, "Decentralized robust adaptive control for attitude synchronization under directed communication topology," Journal of Guidance, Control, and Dynamics, vol. 34, no. 4, pp. 1276-1282, 2011.

[47] L. Zhao, J. Yu, C. Lin, and H. Yu, "Distributed adaptive fixedtime consensus tracking for second-order multi-agent systems using modified terminal sliding mode," Applied Mathematics and Computation, vol. 312, pp. 23-35, 2017.

[48] C. Qian and W. Lin, "A continuous feedback approach to global strong stabilization of nonlinear systems," IEEE Transactions on Automatic Control, vol. 46, no. 7, pp. 10611079, 2001.

[49] L. Yang and J. Yang, "Nonsingular fast terminal sliding-mode control for nonlinear dynamical systems," International Journal of Robust and Nonlinear Control, vol. 21, no. 16, pp. 1865-1879, 2011. 